\title{
Die Elektronische Kirche in den Vereinigten Staaten von Amerika
}

\author{
von Jutta Odile Hess
}

Die Massenmedien in den 80er Jahren reagierten auf den raschen Aufstieg und Popularitätsgewinn religiös-konservativer Fernsehprediger. Ihr sichtbarer wirtschaftlicher Erfolg, ihre politischen Bestrebungen, sowie die Finanz- und SexSkandale einiger populärer Fernsehpfarrer hatten ihren Nachrichtenwert schlagartig erhöht. In den USA wie auch in Deutschland wurden sie von den Medien als „Showmaster im rosa Smoking“ stereotypisiert, ihr Erfolg auf zwielichtige Geschäftspraktiken zurückgeführt. Dies ist zwar auch das Bild, welches bei einer großen Öffentlichkeit in Erinnerung bleibt, doch die Televangelisten und ihre Fernsehkirchen sind auch ein für die Sozialwissenschaften bedeutsames Phänomen.

Angeregt durch die öffentliche Diskussion in den Medien, stieg in den 80er Jahren das wissenschaftliche Interesse an der sog. „elektronischen Kirche" - den unabhängigen, publikumsunterstützten Fernsehsendungen religiös-konservativer Prediger in den Vereinigten Staaten von Amerika.

Doch der Begriff elektronische Kirche beinhaltet mehr als nur religiöses Fernsehen, wie es in geringerem Maße auch von anderen religiösen Gruppen in Amerika produziert wird. Die elektronische Kirche ist vor allem eine Industrie und eine etablierte Institution innerhalb der amerikanischen Religions- und Fernsehlandschaft.

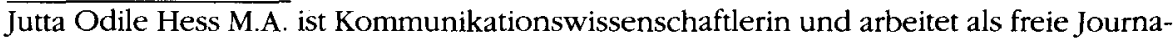
listin in Mainz.

1 Der englische Begriff „electric church“, den Ben Armstrong 1979 mit seinem Buch: The Electric Church. Nashville/New York: Thomas Nelson Publishers, Inc. 1979, prägte, wird in der Literatur von Befürwortern des Phänomens verwendet. Kritiker dagegen sprechen von der "electronic church“, von „pray TV“ oder "the docetic church“. Vgl. Razelle Frankl, Televangelism. The Marketing of Popular Religion. Carbondale: Southern Illinois University Press 1987, 7; Louis Gorfain, Pray TV. In: New York (6. Oktober 1980), 48; Mark R. Sills, The Docetic Church. In: The Christian Century 98 (21. Januar 1981), 37.

In diesem Artikel wird die deutsche Übersetzung „elektronische Kirche" von der Verfasserin ohne negative oder positive Konnotation verwendet. Das hybride englische Wort "televangelism" - 1981 von Jeffrey K. Hadden und Charles E. Swann durch ihr Buch „Prime Time Preachers. The Rising Power of Televangelism“ eingeführt bezeichnet ebenfalls die Fernsehaktivitäten evangelikaler und anderer religiös-konservativer Prediger, wobei dieser Begriff die soziale Bedeutung des Phänomens impliziert. Um auf diese Konnotation hinzuweisen, wird in dieser Arbeit der Begriff nicht übersetzt. Nur für Prediger der elektronischen Kirche, und nicht etwa für religiöse Fernsehveranstalter allgemein, werden im folgenden die Begriffe „Fernsehprediger“, „Televangelisten“, „Fernsehpfarrer“, „Fernsehpastor" und dergleichen verwendet. Auch hier werden keine Konnotationen impliziert. 
Dieser Artikel soll einen Überblick über die elektronische Kirche in den Vereinigten Staaten geben, die es in dieser Form in Deutschland noch nicht gibt. ${ }^{2}$ Da die elektronische Kirche ein integraler Bestandteil des kulturellen, wirtschaftlichen, sozialen und politischen Lebens in Amerika ist, befassen sich Studien über sie, sowohl mit kommunikationswissenschaftlichen als auch mit soziologischen Aspekten.

Es soll dargestellt werden, auf welch spezifische Weise die beiden Komponenten „elektronisch“ (bzw. Fernsehen) und „Kirche“ (bzw. Religion) zum Entstehen und zum Erfolg dieser Institution beigetragen haben. Bereits die ersten puritanischen Siedler Amerikas Anfang des 17. Jahrhunderts und die Erweckungsprediger des 18. und 19. Jahrhunderts haben sowohl die Wert- und Moralvorstellungen als auch die Ziele und Inhalte der elektronischen Kirche bestimmt.

Erläutert wird die Struktur und Organisation der amerikanischen Fernsehindustrie und die Entwicklung des religiösen Rundfunks. Hier haben spezielle Umstände, wie zum Beispiel die Entscheidungen der „Federal Communications Commission" (FCC) und die Regelungen der großen überregionalen Fernsehgesellschaften CBS, NBC und ABC, die besondere Ausformung der elektronischen Kirche entscheidend mitbestimmt.

Ferner soll gezeigt werden, daß die Televangelisten, die man in einem Modell des Kommunikationsprozesses auch als Sender bzw. Kommunikatoren bezeichnen würde, ${ }^{3}$ nicht nur mittels ihrer durch das Fernsehen vermittelten Botschaft eine breite Öffentlichkeit erreichen wollen, sondern auch andere Wege suchen. Hier wird das Ausmaß des Einflußes der konservativen Fernsehprediger auf die Legislative, Exekutive und Judikative im Amerika der 80er Jahre verdeutlicht.

Abschließend folgt eine Analyse der Rezipienten der Televangelisten und ihrer Sendungen. Ein Bild über die Einschaltquoten der elektronischen Kirche, die demographische, sozio-ökonomische und religiöse Zusammensetzung ihrer $\mathrm{Zu}$ schauer, sowie ihre Motivationen, diese religiösen Fernsehsendungen zu sehen, soll es ermöglichen, den Einfluß und die Wirkung der elektronischen Kirche zu ermessen.

Die Betrachtung des gegenwärtigen Forschungsstandes zur elektronischen Kirche zeigt, daß diesem Thema in der Kommunikationsforschung in Deutschland und sogar in Amerika relativ wenig Beachtung geschenkt wurde.

2 Eine ausführlichere Behandlung dieses Themas bietet die Magisterarbeit „Die Elektronische Kirche in den Vereinigten Staaten von Amerika“ von Jutta Odile Hess, erstellt am Institut für Publizistik der Johannes Gutenberg-Universität Mainz vom Juli 1992.

3 Für einen Abriß verschiedener Modelle des Kommunikationsprozesses siehe Elisabeth Noelle-Neumann/Winfried Schulz/Jürgen Wilke (Hg.), Das Fischer Lexikon. Publizistik Massenkommunikation. Frankfurt/M.: Fischer Taschenbuch Verlag GmbH $1989,100-105$. 


\section{Religion und Religiösität in den Vereinigten Staaten}

Keine industrialisierte Nation ist so religiös wie Amerika. Nach einer GallupUmfrage glauben $94 \%$ der Amerikaner an Gott und $40 \%$ der Bevölkerung geht regelmäßig zur Kirche. ${ }^{4}$ Die persönliche, private Religion der Amerikaner spiegelt sich in der für die heutige religiöse Landschaft Amerikas kennzeichnenden Bekenntnisvielfalt wieder. Es gibt über $200 \mathrm{sog}$. Denominationen in den USA, d. h. vom Staat unabhängige religiöse Gruppen, deren Mitgliedschaft und Finanzierung auf dem Prinzip der Freiwilligkeit („voluntarism“) beruht." In den USA werden religiöse Gruppen und Kirchen nicht vom Staat unterstützt, der ihnen lediglich steuerliche Privilegien gewährt. Da auch keine Kirchensteuern erhoben werden, müssen sich die Denominationen durch Spenden ihrer Mitglieder finanzieren. Es ist üblich, daß Gemeindemitglieder ihrer Denomination mindestens ein Zehntel ihres Einkommens abgeben. Diese Finanzierungsmethode ist ein Erklärungsgrund für das Entstehen und den Erfolg der elektronischen Kirche, die hauptsächlich durch Spendeneinnahmen finanziert wird.

Mit etwa $38 \%$ der Gesamtbevölkerung sind die Katholiken die größte Denomination in den USA (circa 50 Mio. Mitglieder). 40 Mio. Amerikaner gehören den sog. weißen "Mainline"-Kirchen an, d. h. den orthodox protestantischen Denominationen - wie Presbyterianer, Episkopalisten, Kongregationalisten, Anglikaner u. a. ${ }^{6}$ Die Mainline-Protestanten werden durch die Dachorganisation "National Council of the Churches of Christ" (NCC) repräsentiert, während die evangelikalen Protestanten in der "National Association of Evangelicals" (NAE) organisiert sind. Etwa $30 \%$ aller Amerikaner bekennen sich zu den Evangelikalen, unter Einschluß der Baptisten und der diversen fundamentalistischen Gruppen (etwa 40 Mio. Mitglieder). Amerikaner jüdischen Bekenntnisses machen etwa 3\% der Gesamtbevölkerung aus.?

Der Protestantismus der Gegenwart läßt sich in ein liberales, ein gemäßigtes und ein konservatives Lager unterteilen. Als liberale Protestanten gelten z. B. die Episkopalisten, die Presbyterianer und die United Church of Christ. Sie stellen etwa $18 \%$ der gesamten protestantischen Bevölkerung Amerikas dar. Zu den gemäßigten Protestanten zählen die sog. „frontier"-Religionen - die Methodisten, die Baptisten des Nordens und die Disciples of Christ - und die mittel- und nordeuropäischen Einwandererreligionen: die Vereinigten Lutheraner und die holländisch und deutsch Reformierten. Ihr Anteil am amerikanischen Protestantismus beträgt ca. 44 $\%$ verglichen mit $38 \%$ konservativen Protestanten, zu denen unter anderen die

4 In den meisten europäischen Ländern liegt die entsprechende Quote unter $8 \%$. Vgl. Erich Geldbach, Religion und Politik: Religious Liberty. In: Klaus-M. Kodalle (Hg.), Gott und Politik in den USA. Frankfurt/M.: Athenäum Verlag 1988, 238.

5 Die Denomination ist die rechtliche Organisationsform religiöser Gruppen in den USA. Vgl. Linda K. Pritchard, Religious Change in Nineteenth-Century America. In: Charles Y. Glock/Robert N. Bellah (eds.): New Religious Consciousness. Berkeley/ Los Angeles/London: University of California Press 1976, 303.

6 Vgl. Wade Clark Roof, America's Voluntary Establishment: Mainline Religion in Transition. In: Daedalus, Vol. 111 (Winter 1982), 171.

7 Vgl. Jeffrey K. Hadden/Charles E. Swann, Prime Time Preachers. The Rising Power of Televangelism. Massachusetts/California/etc.: Addison-Wesley Publishing Company, Inc. 1981,77 
Baptisten des Südens, Evangelikale und Fundamentalisten und die charismatischen Bekenntnisse - die Pentecostals, Holiness-Gruppen, die Assemblies of God, die Churches of God - zählen.

Als Evangelikale bezeichnen sich in den Vereinigten Staaten Mitglieder verschiedener, vorwiegend protestantischer Denominationen. Trotz unterschiedlicher theologischer Auffassungen zwischen den Evangelikalen verschiedener Denominationen gibt es einige gemeinsame Merkmale, wie beispielsweise die verpflichtende Bindung an die Bibel als das Wort Gottes, die Vergebung der Sünden der Menschen nur durch den Glauben (nicht durch eigene Wiedergutmachung) und die Notwendigkeit des persönlichen Bekehrungserlebnisses (nur der kann sich als Christ bezeichnen, der sich in einer bestimmten Form persönlich für das Heilsangebot Gottes entschieden hat, d. h. die Erfahrung der „Wiedergeburt aus dem Glauben" gemacht hat). Damit verbunden ist ein Bemühen um Einheit aller "wiedergeborenen" Christen sowie die Bereitschaft, sich persönlich in Evangelisation und Mission zu engagieren.

Der Evangelikalismus entwickelte sich aus den Erweckungswellen des 19. Jahrhunderts. Repräsentativ für die Institutionalisierung dieser Erweckungsbewegungen stehen drei Evangelisten - Charles G. Finney, Dwight L. Moody und Billy Sunday. Ihre zwischen 1830 und 1900 initiierten Evangelisationskampagnen waren perfekt organisierte und geplante Massenveranstaltungen. Durch die Verteilung von Flugblättern sowie Zeitungs- und Plakatwerbungen sicherten sich diese Laienpfarrer eine große Teilnahmerzahl an ihren Evangelisationsveranstaltungen. Sie setzten ferner neue Methoden der rationalen und logischen Überzeugungskunst (Persuasion) ein und machten aus den mystischen, spiritistischen Erweckungen pragmatische, routinierte Ereignisse. Um bei den Massen religiösen Enthusiasmus zu schüren, predigten sie eine populär inspirierende Religion, wobei sie dramatische, umgangssprachliche und emotionale Rhetorik verwendeten. Auch persönliche Ansprachen an zu bekehrende Personen und der Einsatz von populären musikalischen Elementen, die vom Publikum mitgesungen werden konnten, gehörten zum Repertoire dieser Erweckungsprediger. ${ }^{8}$

Finney, Moody und Sunday trugen zur Demokratisierung des amerikanischen Protestantismus bei, indem sie statt der calvinistischen Prädestinationslehre eine Theologie der individuellen moralischen Verantwortung und des aktiven Evangelikalismus vertraten. Nicht nur auserwählte Personen, sondern jeder aktiv Gläubige konnte die Gnade Gottes erlangen. ${ }^{9}$ Mit dieser neuen Ethik gingen strukturelle Veränderungen einher: Im Mittelpunkt standen nicht mehr die Pfarrer der herkömmlichen protestantischen Kirchen, die durch ihre verurteilenden und drohenden Predigten Autorität erlangt hatten, sondern die Erweckungsprediger, die mit ihren rhetorisch einfach aufgebauten und hoffnungsspendenden Predigten

8 Vgl. Jeffrey K. Hadden/Anson D. Shupe (eds.), Televangelism. Power and Politics on God's Frontier. New York: Henry Holt and Company, 1988, 44; Larry Martz/Ginney Carroll (eds.), Ministry of Greed. The Inside Story of Televangelists and Their Holy Wars. A Newsweek Book. New York: Weidenfeld and Nicolson, 1988, 28.

9 Vgl. Razelle Frankl, Televangelism (Anm. 1), 36, 41; Larry Martz/Ginney Carroll (eds.), Ministry of Greed (Anm. 8), 27, 28; Jeffrey K. Hadden/Anson D. Shupe (eds.), Televangelism (Anm. 8), 44. 
ihren Anhängem den Prozeß der Erlösung erleichtern wollten. Die neue Rolle des Predigers war die des Vermittlers.

Diesen Predigern wurde, wie später den Fernsehpredigern, der Vorwurf des Anti-Intellektualismus gemacht, d. h. der Geringschätzung theologischer Probleme bei gleichzeitiger Förderung einer passiven "pop culture“ der Massen durch unterhaltsame Veranstaltungen. ${ }^{10}$

Auch setzten Finney, Moody und Sunday neue Maßstäbe für Erfolg und Weisheit: Nicht die Ausbildung und der soziale Status eines Pfarrers innerhalb seiner Kirche, sondern nur die Zahl der bekehrten Seelen, die die nötigen finanziellen Ressourcen stellten, galten als Erfolgskriterium. Diese christliche Verkaufsstrategie ist heute kennzeichnend für die elektronische Kirche. Wegen der Abhängigkeit und der Fixierung auf statistische Erfolgsnachweise propagieren die Televangelisten einen entmystifizierten Gott des positiven Denkens, der Christen aller Denominationen und gesellschaftlicher Schichten anspricht. Spendern werden im Gegenzug Seelenheil und Geschenke versprochen. ${ }^{11}$

Moody und Sunday umgingen die Autoritätsstrukturen der lokalen Kirchengemeinden und wandten sich an prominente Geschäftsleute mit der Bitte um finanzielle Unterstützung ihres Vorhabens. Damit schufen diese Erweckungsprediger erstmals evangelikale Imperien, denominationell unabhängige, sich selbst verwaltende und selbst finanzierende Institutionen. ${ }^{12}$ Finney, Moody und Sunday hatten eine ideale Organisationsform geschaffen, die unabhängig von herkömmlichen Denominationen ihre eigenen Strukturen und Funktionen vorgab. Eine neue aktivitätsorientierte „believe-as-you-like, worship-as-you-please“-Religion brachte einen entmystifizierten Gott hervor, der der Allgemeinheit nützlich sein sollte. ${ }^{13}$ Der charismatische Prediger verkörperte eine neue utilitaristische Ethik, die einen populären, inspirierenden und unterhaltsamen Predigerstil sowie den Einsatz neuer psychologischer Methoden, Geschäftspraktiken und moderner Kommunikationsmittel zur Mobilisierung eines großen Publikums rechtfertigte. Mit ihrer individualistischen, anti-autoritären, populistisch-demokratischen Grundhaltung können die Erweckungsprediger des 19. Jahrhunderts als Vorläufer der Fernsehevangelisten des späten 20. Jahrhunderts gesehen werden. Sie schufen mit ihren parakirchlichen Unternehmen die institutionelle Grundlage für die elektronische Kirche der 70er und 80er Jahre, die als Teil einer traditionalistischen Bewegung konservativer Protestanten innerhalb einer neuen Erweckungsperiode gesehen werden kann.

Aus dem durch die Erweckungsbewegungen entstandenen Evangelikalismus entwickelte sich Anfang des 20. Jahrhunderts die konservative Bewegung des Fundamentalismus als eine Art „reaktionärer Evangelikalismus“ gegen die sozialreformerische „Social Gospel“-Theologie und die moderne Bibelkritik der liberalen Theologie.

10 Vgl. Razelle Frankl, Televangelism (Anm. 1), 14, 30.

11 Vgl. Klaus-M. Kodalle, Zivilreligion in Amerika: Zwischen Rechtfertigung und Kritik. In: Klaus M. Kodalle (Hg.): Gott und Politik in den USA. Frankfurt/M.: Athenäum Verlag, 1988, 19-73, 49ff.

$12 \mathrm{Vgl}$. Razelle Frankl, Televangelism (Anm. 1), xiv, 46-47.

13 Vgl. Klaus-M. Kodalle (Anm. 11), 49. 
Der persönlichen, privaten Religion der Amerikaner steht eine öffentliche, altgemeine Religion, auch „Zivilreligion“ genannt, gegenüber. ${ }^{14}$ Die Zivilreligion ist an keine einzelne Religion gebunden, sondern integriert politische und religiöse Ideen der amerikanischen Geschichte - wie das puritanische Sendungsbewußtsein, den Missionsgedanken der Erweckungsprediger, das patriotistische und zugleich pragmatische Denken konservativer Protestanten - zu einer spezifischen Symbolik. Die Bezugnahme auf diesen zivilreligiösen Symbolkomplex dient der Konsensbildung zwischen den verschiedenen Ethnien und Religionen Amerikas. Anhänger der Neuen Christlichen Rechten und die religiös-konservativen Fernsehprediger der elektronischen Kirche hatten seit Ende der 70er Jahre eine Artikulationsfunktion für die rhetorischen Muster des wertkonservativen amerikanischen Credos.

\section{Die Fernsehindustrie und religiöses Fernsehen in den Vereinigten Staaten}

\subsection{Die Fernsehindustrie}

Die zunehmende Zahl von Fersehstationen in Amerika ${ }^{15}$, die hohe Anzahl von Fernsehgeräten in amerikanischen Haushalten ${ }^{16}$ und die starke Nutzung des Mediums ${ }^{17}$ lassen sich durch den privatwirtschaftlich-kommerziellen Charakter des Rundfunks in Amerika erklären.

1920 ging der erste kommerzielle amerikanische Radiosender auf Sendung. 1926 gab es in den USA bereits 500 Sender, die rein privatwirtschaftlich waren, d. h. unabhängig von staatlichen Instanzen. Sie finanzierten sich nur durch den Verkauf von Sendezeiten. ${ }^{18}$

14 Vgl. Robert N. Bellah, Civil Religion in America. In: Daedalus, Vol. 96 (1967), 1-21; Jürgen Gebhardt, Amerikanismus - Politische Kultur und Zivilreligion in den USA. In: Aus Politik und Zeitgeschichte. Beilage zur; Wochenzeitung Das Parlament B49/90 (30. November 1990), 16f.; Gustav H. Blanke, Das amerikanische Sendungsbewußtsein: Zur Kontinuität rhetorischer Grundmuster im öffentlichen Leben in den USA. In: Klaus-M. Kodalle (Hg.), Gott und Politik in den USA. Frankfurt/M.: Athenäum Verlag 1988, 187-188; Klaus-M. Kodalle, Zivilreligion in Amerika, (Anm. 11), 19-73.

151981 gab es 1031 kommerzielle Fernsehsender und 269 öffentliche, nicht kommerzielle Sender. Vgl. Tom Bethell, Spreading the News. In: Mal Oettinger/John Stirn/ Valerie Kreutzer (eds.): The Role of the Media. USA: United States Information Agency, o.J. The Role of the Media, 20.

16 Statistiken zeigen, daß fast jeder Haushalt ein oder mehrere Geräte besitzt. Vgl. Ben Armstrong, The Electric Church (Anm. 1), 82; George Comstock, The Impact of Television on American Institutions. In: JOC 28 (Frühling 1978), 12

17 Nach einer Statistik des Forschungsinstituts A.C.Nielsen Company von 1985 läuft der Fernsehapparat durchschnittlich sieben Stunden am Tag, wobei der Durchschnittsamerikaner durchschnittlich viereinhalb Stunden am Tag bzw. 30 Stunden pro Woche fernsieht. Sendungen mit den höchsten Einschaltquoten erreichen bis zu 36 Millionen Haushalte. Vgl. William Fore, Television and Religion, The Shaping of Faith, Values and Culture. Minneapolis: Augsburg Publishing House 1987, 16.

18 Vgl. Joachim Schmidt, Rundfunkmission - ein Massenmedium wird Instrument. Erlangen: Verlag der Ev.-Luth. Mission, 1980, 32. 
Fast jeder Antragsteller erhielt vom amerikanischen Handelsministerium („U.S. Department of Commerce“) eine Sendefrequenz. Bald kam es zu Störungen und Überlagerungen in den Luftwellen, was die amerikanische Regierung veranlaßte, eine staatliche Reglementierung im technischen Bereich einzuführen. Der Kongreß verabschiedete das ,Radio Act of $1927^{\circ}$ und erteilte der „Federal Radio Commission“ (FRC), aus der 1934 die „Federal Communications Commission“ (FCC) hervorging, die alleinige Befugnis der Frequenzenverteilung und Fernsehlizenzerteilung. ${ }^{19}$

Die „Federal Communications Commission“ (FCC) ist eine dem amerikanischen Kongress direkt unterstellte, aber regierungsunabhängige Regulierungskommission. ${ }^{20}$ Die FCC unterscheidet zwischen den „kommerziellen“ Sendern dazu gehören alle privatwirtschaftlichen, durch Werbung finanzierten, regionalen und lokalen Rundfunk- und Fernsehgesellschaften, d. h. auch viele religiöse Fernsehsender - und den öffentlichen, „nicht-kommerziellen" Sendern, die von Gemeinden, politischen und kulturellen Verbänden, Universitäten und ähnlichen Institutionen betrieben und durch Zuschauerspenden und jährliche Bundeszuschüsse unterstützt werden. Die Richtlinien zur Regulierung kommerzieller und nichtkommerzieller Fernsehstationen unterscheiden sich allerdings kaum. Der wesentliche Unterschied besteht in der Tatsache, daß nicht-kommerzielle Sender keine Werbezeiten verkaufen dürfen.

Ausgeschlossen von diesem Lizenzierungssystem sind die sog. „networks“, die großen nationalen Rundfunkanstalten in den USA, wie beispielsweise NBC, ABC und CBS. Sie werden als „program distribution companies“ angesehen, $d$. $h$. sie kaufen auf vertraglicher Basis Sendungen von freien Fernsehproduktionsfirmen und liefern diese an ihre im ganzen Land vertretenen Tochtergesellschaften, die sog. „affiliates“. Der 1934 gegründeten FCC steht gemäß dem „Communications Act" von 1934 nur die öffentliche Überwachung technischer Vorschriften im Rundfunk zu und nicht die Einflußnahme auf die Programmgestaltung, d. h. die Behörde hat keine Möglichkeiten der Einflußnahme (z. B. durch Zensur) auf Qualität und Quantität der Programme und auf die Werbung. Doch da der kommerzielle Rundfunk als öffentlicher Besitz angesehen wird, müssen sich diejenigen, die ihn nutzen, auch gegenüber der Öffentlichkeit in einer bestimmten Weise verpflichten. Die Vergabe oder Erneuerung einer Sendelizenz hängt davon ab, ob der Bewerber sich bereit erklärt Programme anzubieten, die dem öffentlichen Interesse (,public interest") dienen. ${ }^{21}$

Die Art der Finanzierung der kommerziellen Rundfunk- und Fernsehgesellschaften Amerikas durch Werbeeinnahmen, beeinflußt die Programmgestaltung und den Inhalt der Sendungen. Die Abhängigkeit vom Verkauf von Werbezeiten und damit von steigenden Zuschauerzahlen führt zu einer Homogenisierung der Programmgattungen, einer Nivellierung der Inhalte und zu zunehmender Syndikalisierung von Programmen. Dies erklärt den großen Anteil von Unterhaltungssen-

12 Vgl. Quentin J. Schultze, Evangelical Radio and the Rise of the Electronic Church, 1921-1948. In: Journal of Broadcasting \& Electronic Media 32/3 (1988), 292.

$20 \mathrm{Vgl}$. Tom Bethell, Spreading the News (Anm. 15), 18.

21 Vgl. Linda Jo Lacey, The Electric Church: An FCC-"Established" Institution? In: Federal Communications Law Journal 31 (Frühling 1979), 247 
dungen und Serien im amerikanischen Fernsehen und die Herausbildung eines "Prominenten-Systems" im Unterhaltungsbereich, bei den Nachrichten sowie bei der elektronischen Kirche. Dadurch wird den Zuschauern die Identifikation mit dem Programm erleichtert, um eine Bindung an das Programm zu erreichen. Schließlich macht sich die Abhängigkeit von den Gesetzen des kapitalistischen Marktes auch in der Struktur des Programms bemerkbar, welches in fünfzehn-, dreißig- und sechzigminütige Einheiten aufgeteilt ist.

Die Konkurrenz der überregionalen Sendeanstalten NBC, ABC und CBS, die in den 70er Jahren etwa $90 \%$ und 1990 nur noch $66 \%$ der Fernsehzuschauer an sich binden konnten, sind etwa 5.000 Kabelsysteme in den USA. Zwei Drittel der ca. 90 Mio. Fernsehhaushalte waren 1990 verkabelt und konnten bis zu 50 Kanäle empfangen. Das Programm der Kabelsender wird teilweise selbst produziert und teilweise von syndikalisierten Satellitenprogrammen übernommen. ${ }^{22}$

\subsection{Religiöses Fernseben}

Amerikanische Kirchen und Denominationen reagierten sofort auf das neue Medium Radio: Schon 1920, dem Jahr der ersten kommerziellen Rundfunkübertragung, wurde bereits der erste Gottesdienst ausgestrahlt. Bald schon kauften und betrieben religiöse Organisationen ihre eigenen meist nicht-kommerziellen Sender. 1924 betrieben lokale Kirchengemeinden und andere religiöse Organisationen 29 Stationen und 1925 stieg die Zahl auf $71 .^{23}$

Zwar erhielten Anfang der 20er Jahre Mainline-Kirchen und evangelikale Gruppen in ausgewogenem Maße Lizenzen vom amerikanischen Handelsministerium, doch evangelikale Protestanten, insbesondere Baptisten, dominierten im religiösen Rundfunk. Dies lag einerseits an dem geistigen Klima der Zeit, welches von einem geschwächten traditionellen Protestantismus und einem durch die Erweckungsbewegungen erstarkten Evangelikalismus gekennzeichnet war. Andererseits bedingte das Aktivitätsprinzip der evangelikalen Denominationen - verbunden mit dem Erfolgszwang, der aus der calvinistischen Tradition herrührte, und der Adaption an den "American Way of Life“ - unterschiedliche Einstellungen gegenüber der Übernahme dieser neuen Methoden zur Verkündigung religiöser Botschaften: Während die Verwendung des neuen Mediums die skeptischen Mainline-Kirchen zu theologischen Reflexionen bewegte, sahen die Evangelikalen im Rundfunk eine neue Möglichkeit, die institutionalisierten Erweckungsbewegungen des 19. Jahrhunderts fortzuführen.

Die rapide Zunahme evangelikaler Radioprediger wurde durch das neue Radio-Gesetz von 1927 und die damals vom Staat neu gebildete „Federal Radio Commission“ (FRC) gebremst. Religiösen Gruppen wurden von der FRC keine neue Sendelizenz erteilt und bestehende religiöse Sender wurden gezwungen, entweder ihre Frequenzen mit anderen Sendern zu teilen oder eine Frequenz nur

22 Vgl. Berndt Ostendorf, Radio und Fernsehen. In: W. P. Adams/E.-O. Czempiel/B. Ostendorf u. a. (Hg.): Länderbericht USA II, Bonn: Bundeszentrale für politische Bildung, 1990, 589.

23 Vgl. Quentin J. Schultze, Evangelical Radio and the Rise of the Electronic Church (Anm. 19), 291. 
zeitlich begrenzt zu nutzen. Zwar reduzierte sich die Zahl religiöser Radiosender in den 30er Jahren, doch die Evangelikalen und Fundamentalisten wandten sich nun an kommerzielle Stationen in ganz Amerika, um ihr Programm zu verbreiten. Mit der Entwicklung der "networks" war es nun möglich, mit einer Predigt etwa eine Million Menschen zu erreichen.

Die Regelungen der "Federal Communications Commission“ enthielten bis 1960 eine Klausel, wonach Rundfunkanstalten gemeinnützigen Organisationen ("non-profit organizations") - dazu gehörten auch Kirchen und Denominationen einen gewissen Prozentsatz an kostenloser Sendezeit zur Verfügung stellen mußten, um ihr Quantum an „public interest“ zu erfüllen. Es war den Rundfunkanstalten überlassen, religiösen Gruppen kostenlose Sendezeit ( „free“ oder "sustaining time“) zur Verfügung zu stellen oder sie zu verkaufen („paid“ oder „commercial time) ${ }^{24}$ Wenn kostenlose Sendezeiten vergeben wurden, dann meistens nur an die gut organisierten Denominationen, d. h. an die Katholiken, an die Mainline-Protestanten mit ihrer Organisation „Federal Council of Churches of Christ“ (FCCC) und zu einem geringeren Teil an die Juden.

Die Evangelikalen dagegen erhielten selten kostenlose Sendezeiten. So kam es, daß viele evangelikale Prediger, aufgrund der ihnen zugeteilten schwachen Sendefrequenzen, in den 30er Jahren ihre Radiostationen aufgaben und mit Hilfe der eingenommen Spendengelder Sendezeiten bei kommerziellen lokalen Radiosendern, u. a. auch bei einigen"affiliates" der nationalen Rundfunkanstalten kauften.

Dieser offensichtliche Nachteil für die evangelikalen Radioprediger wirkte sich allerdings für den Aufbau der elektronischen Kirche positiv aus, denn die Evangelikalen meisterten bald die Produktion von publikumsattraktiven Sendungen und die Mobilisierung von Spendengeldern.

1932 strahlten die Evangelikalen bereits mehr als 400 religiöse Programme auf 80 lokalen Sendern aus - damit bestritten sie mehr als $8 \%$ des gesamten nationalen Radioprogramms. ${ }^{25}$ Evangelikale Baptisten, Fundamentalisten und Anhänger der Pfingstkirchen, die zwei Drittel ihrer Sendezeiten kauften, hatten insgesamt mehr Übertragungszeit als Mainline-Protestanten, die nur ein Drittel ihrer Zeit kaufen mußten.

Um die Evangelikalen auf den lokalen Rundfunk zu beschränken, versuchten die überregionalen Fenrsehanstalten und die Mainline-Protestanten, vertreten durch die FCCC, durch eine restringierende Politik, evangelikale Rundfunkgestalter aus dem nationalen Rundfunkbetrieb auszuschließen.

1942 gründeten die konservativen Christen die „National Association of Evangelicals" (NAE), um sich gegen den Einfluß der "Federal Council of Churches" (FCCC) zu behaupten. ${ }^{26} 1944$ gründeten dann 150 evangelikale Funk- und Fern-

24 Vgl. Razelle Frankl, Televangelism (Anm. 1), 68.

25 Vgl. Jeffrey K. Hadden/Anson D. Shupe (eds.), Televangelism (Anm. 8), 113; Quentin J. Schultze, Evangelical Radio and the Rise of the Electronic Church (Anm. 19), 296.

261979 vertrat die "National Association of Evangelicals“ (NAE) mehr als 38.000 konservative Kirchen in Amerika. Vgl. Ben Armstrong, The Electric Church (Anm. 1), 49. 
sehmacher der NAE eine eigene Organisation für evangelikale Rundfunkbetreiber - die "National Religious Broadcasters" (NRB). Religiöse Organisationen traten 1944 der NRB bei, 1985 waren bereits 1050 evangelikale Rundfunkbetreiber Mitglieder.

Die NRB setzte sich für die Rede- und Meinungsfreiheit im religiösen Rundfunk ein und für die uneingeschränkte Freiheit, Sendezeiten für religiöse Programme zu kaufen. Ihre Bemühungen führten zu einigen Erfolgen für den evangelikalen Rundfunk. Trotzdem war es für konservative Christen in den 40er und 50er Jahren schwer, sich in dem neuen Medium Fernsehen gegen die Mainline-Kirchen zu behaupten, die in dieser Zeit den Höchststand ihrer Mitgliederzahlen erlebten. Die Mainline-Protestanten, die zum „mainstream“ des amerikanischen kulturellen und religiösen Lebens gehörten, waren damit auch stärker in der Fernsehindustrie vertreten als die Evangelikalen oder Fundamentalisten, die darauf kaum einen Einfluß hatten. Erschwerend kam hinzu, daß sich die liberalen Protestanten 1950 in der „National Council of Churches of Christ“ (NCC) neu organisiert hatten. Die NCC hatte ebenfalls eine eigene Abteilung für religiösen Rundfunk gegründet - die „Broadcasting and Film Commission" (BFC) -, die erneut gegen die bezahlten religiösen Programme vorging. Sie drängten die nationalen Sendeanstalten „sustained-time-only"-Regelungen für das Radio und das neue Medium Fernsehen zu erstellen. ${ }^{27}$

Das dualistische System - kostenlose Sendezeit bei den überregionalen Sendeanstalten für die organisierten Kirchen, insbesondere die Mainline-Protestanten, und bezahlte Programme bei unabhängigen Stationen für die evangelikalen und fundamentalistischen Anbieter - wurde also vom Radio auf das Medium Fernsehen übertragen. ${ }^{28}$

Dagegen protestierten die evangelikalen Rundfunkgestalter der NRB solange, bis die „Federal Communications Commission“ (FCC) mit dem "Code of Standards of $1960^{\prime \prime}$ entschied, daß nicht nur kostenlos gesendete kirchliche Programme, sondern auch bezahlte religiöse Sendungen das Kriterium der "public interest" erfüllen müssen.

Es war natürlich viel lukrativer, Geld für Sendezeiten einzunehmen als die Produktionskosten für die Mainline-Programme zu stellen. Daher kamen die lokalen Sender, die sich bis zu diesem Zeitpunkt meistens an die Vorgaben der nationalen Rundfunkanstalten gehalten hatten,den evangelikalen und fundamentalistischen Predigern, die bereit waren ihre Sendezeiten zu kaufen, immer mehr entgegen. Der zunehmende Wettbewerb unter Fernsehevangelisten führte zu einer Steigerung des Verkaufswertes der Sendeplätze.

Während Anfang der 60er Jahre die Hälfte aller religiösen Fernsehprogramme von den etablierten Kirchen und die andere Hälfte von evangelikalen Denominationen bestritten wurden, änderte sich diese Gewichtung noch im Laufe des Jahrzehnts. Die Zahl der kostenlos gesendeten Programme der großen Rund-

27 Vgl. Jeffrey K. Hadden/Anson D. Shupe (eds.), Televangelism (Anm. 8), 49.

28 Vgl. Stewart M. Hoover, The Electronic Giant: A Critique of the Telecommunications Revolution from a Christian Perspective. Illinois: The Brethren Press, 1982, 119. 
funkanstalten ging stark zurück, während die Zahl der denominationell unabhängig produzierten, durch Zuschauerspenden finanzierten religiösen Programme und Stationen, die den Namen elektronische Kirche erhielten, deutlich anwuchs. 1970 war die elektronische Kirche der am schnellsten expandierende Sektor des amerikanischen Fernsehens. Diese Entwicklung, die einherging mit Mitgliederverlusten der Mainline-Kirchen und dem Erstarken der konservativen Christen, beschleunigte sich im Laufe der 70er Jahre und flachte erst nach den Skandalen der Televangelisten Jim Bakker und Jimmy Swaggart Mitte der 80er Jahre ab.

Aufgrund nachteiliger Behandlung durch die FCC und die nationalen Rundfunkanstalten waren die evangelikalen Prediger schon früh darauf angewiesen, Sendestunden beim kommerziellen Rundfunk zu erwerben. Als Folgeerscheinung erlangten sie technische Professionalität und übten sich in der Herstellung publikumsattraktiver Religionsunterhaltung, um die Spendeneinnahmen durch ihre Zuschauer zu sichern. Die Mainline-Kirchen hingegen kamen selten über „langweilige Abfilmungen sonntäglicher Gottesdienste" hinaus. ${ }^{29}$

Wie das evangelikale Radio in den 20er und 30er Jahren, so wurde nun die elektronische Kirche zum ,big business', das gleichzeitig zur Integration der evangelikalen Bewegung beitrug und ihr eine nationale Identität verlieh. Die elektronischen Medien ermöglichten es den konservativen Christen Amerikas, ihre eigenen charismatischen Führer zu fördern und ihre Wertvorstellungen zu legitimieren.

\section{Die Elektronische Kirche bzw. das Phänomen des „Televangelism“}

Die elektronische Kirche bezeichnet nicht religiösen Rundfunk allgemein, sondern die unabhängig produzierten, durch Spendengelder finanzierten und national verbreiteten Programme konservativer Fernsehprediger und ihrer parakirchlichen Unternehmen. ${ }^{30}$ Den Begriff "electric church“ für diese spezielle Form des religiösen Fernsehens prägte 1979 Ben Armstrong, der damalige Direktor der „National Religious Broadcasters" (NRB). ${ }^{31}$

Ben Armstrong argumentiert, die elektronische Kirche sei kein neues Phänomen, sondern nur eine revolutionäre neue Form des rituellen kirchlichen Gottesdienstes mit Einsatz modernster Technologie.

Dagegen vertritt die Soziologin Razelle Frankl die Meinung, daß die elektronische Kirche eine neue sozio-religiöse Institution sei, hervorgegangen aus einer Mischung zwischen den institutionalisierten Erweckungsbewegungen des 19. Jahrhunderts und der Institution des amerikanischen Fernsehens.

29 Hans Norbert Janowski (Hg.): Die kanalisierte Botschaft. Religion in den Medien Medienreligion. Gütersloh: Gütersloher Verlagshaus Gerd Mohn, 1987, 70.

30 Die hier genannte Definition der elektronischen Kirche ist die gängigste. Vgl. Peter G. Horsfield, Religious Television. The American Experience, New York/London: Longman Inc., 1984, 53. Einige wenige Autoren beziehen sich mit diesem Begriff nur auf die 10 populärsten Televangelisten und ihre Fernsehkirchen, andere wiederrum schließen jegliche elektronische Kommunikation ein, deren Inhalt von Sendern und Empfängern gleichermaßen als religiös empfunden wird. Vgl. Razelle Frankl, Televangelism (Anm. 1), 3, 7 .

31 Vgl. Jeffrey K. Hadden/Charles E. Swann, Prime Time Preachers (Anm. 7), 75, $178 f$. 
„Statt eine erfolgreiche Anwendung und Ausdehnung des religiösen Rundfunks zu sein, wird augenscheinlich, daß wir einen völlig neuen Typus ,Kirche haben, eine neue Institution, stimuliert und kreiert mit Hilfe der Fernsehtechnologie. ${ }^{\text {"32 }}$

Razelle Frankl zeigt, daß die elektronische Kirche ein wichtiges Kriterium nicht erfüllt: Die Bildung von Gruppensolidarität und die Integration der einzelnen Gruppenmitglieder in eine rituelle Gemeinschaft. Statt interpersoneller Kommunikation zwischen Pfarrer und Gemeinde tritt die einseitige, unpersönliche Vermittlung religiöser Botschaften durch das Medium Fernsehen. ${ }^{33}$

Martin E. Marty, lutheranischer Pfarrer und Professor an der Universität von Chicago, nennt folgende wesentliche Merkmale der elektronischen Kirche: Sie ist eine Erscheinung konservativer Christen in Amerika und ihrer Rundfunkorganisation „National Religious Broadcasters“ (NRB). Im Mittelpunkt steht ein evangelikaler Prediger, dessen Anziehungskraft nicht nur auf seiner Fähigkeit zu unterhalten basiert, er ist eine Autoritätsperson, dessen Anerkennung durch das Publikum im wesentlichen auf seiner Präsenz durch das Fernsehen beruht. Dadurch ist dieses Publikum - welches Marty bewußt Klientel statt Kirchengemeinde nennt - bereit, ihn und seine Vorhaben durch regelmäßige Spenden zu finanzieren.

„Die sichtbarsten Führer der modernen amerikanischen Religion sind die ,Medienberühmtheiten', die Unterhalter, deren ,Führerschaft' primär auf ihrem Massenanklang beruht. ... Solch ein Führer braucht keine theologische Ausbildung und formale Schulung und Erfahrung in einem geistlichen Amt mehr, weil seine Autorität auf Erfolg - gleich auf welchem Gebiet - beruht."34

Die elektronische Kirche stellt mehr dar als religiöses Fernsehen, sie ist ein unabhängiges Großunternehmen innerhalb der amerikanischen Fernseh- und Religionslandschaft. Wie die Erweckungsprediger Finney, Moody und Sunday, so haben auch die Fernsehprediger der elektronischen Kirche des späten 20. Jahrhunderts kirchen- und staatsunabhängige autarke Organisationen, sog. Parakirchen („parachurches"), geschaffen. Sie basieren auf einer hierarchisch strukturierten Oligarchie, die den Prinzipien der freien Marktwirtschaft gehorcht. Ihre Position innerhalb der amerikanischen Fernsehindustrie ist einzigartig. Keine bürokratischen Strukturen schränken die Macht des Fernsehpredigers ein. Der Gesamtumsatz der 8-10 prominentesten Fernsehprediger Amerikas in den 80er Jahren wurde auf eine Milliarde Dollar geschätzt. 1980 erzielte Jim Bakker beispielsweise 51 Mio. Dollar an Spendeneinnahmen, Pat Robertson 47 Mio. und Jimmy Swaggart 20 Mio. Dollar. Mit diesen Einnahmen finanzierten sie ihre eigenen Universitäten, Zeitungen und Verlage, Schallplattenfirmen für Gospel-Musik und ständige „Projekte“, wie Kran-

32 Von der Verfasserin ins Deutsche übersetzt. Für Originalzitat siehe Razelle Frankl, Televangelism (Anm. 1), 8.

33 Vgl. William F. Fore,'There is no such thing as a TV pastor'. In: TV Guide (19. Juli 1980), 15-18.

34 Von der Verfasserin übersetzt. Für Originalzitat siehe Richard Quebedaux, By What Authority. The Rise of Personality Cults in American Christiänity. San Francisco: Harper \& Row, Publishers, 1982, 113, 114. 
kenhäuser, gläserne Kathedralen, Luxushotels, Freizeitparks etc. Die Wall Street Journal bezeichnete 1978 die elektronische Kirche als „big business“. ${ }^{35}$

Doch im Gegensatz zu anderen wettbewerblichen Unternehmen genießt die elektronische Kirche - wie alle gemeinnützigen und religiösen Organisationen in Amerika - den Status der Steuerbefreiung. Nach den Richtlinien der "Federal Communications Commission" und dem Bundessteuergesetz wird die elektronische Kirche als „religious non-profit organization" klassifiziert. Das bedeutet, daß sämtliche Einnahmen, auch Zuschauerspenden, nicht versteuert werden. Ob die Fernsehevangelisten die Bedingungen einer steuerbefreiten „non-profit“-Organisation erfüllen, überprüft das Bundesfinanzamt der Vereinigten Staaten (IRS). Die amerikanische Börsenaufsichtsbehörde („Securities and Exchange Commission (SEC)) hat die Aufgabe, die Methoden der Kapitalbeschaffung und den Verkauf von Aktien und Wertpapieren durch die Fernsehprediger zu kontrollieren.

Als Ergebnis eines Rationalisierungsprozesses besitzt die elektronische Kirche ihre eigenen Strukturen, Funktionen, Rollen, Normen, Verhaltensmuster und eine eigene Ethik zur effektiven Verfolgung festgesetzter Zielvorstellungen. Zu den Zielvorstellungen der Fernsehprediger gehört die Evangelisierung möglichst vieler Menschen und die Revitalisierung einer sog. „old-time religion“ . ${ }^{36}$

Diese Abhängigkeit von den Zuschauern und deren finanzieller Unterstützung ist einer der wesentlichen Unterschiede zwischen der elektronischen Kirche und dem religiösen Rundfunk anderer Kirchen, wie beispielsweise dem der Mainline-Protestanten. Um die erwünschten Massen zu erreichen und zu binden, predigen die evangelikalen, fundamentalistischen und besonders die charismatischen Fernsehprediger eine populär inspirierende Religion, die mit ihren zivilreligiösen Elementen eine breite Masse von Amerikanern anspricht und die unmittelbare Befriedigung der Individuen verspricht. Damit läßt sich die elektronische Kirche einerseits in die religiöse Tradition der Erweckungsbewegungen in Amerika einordnen, andererseits in die säkulare Tradition der "popular culture“ ${ }^{47}$

Mehr noch als die Anpassung der Programminhalte und -formen an die Bedürfnisse der Durchschnittsamerikaner kennzeichnet die elektronische Kirche die Abhängigkeit von ihren Zuschauern. Um diese an ihr Programm zu binden bzw. eine Produktbindung zu schaffen und die finanzielle Unterstützung zu sichern, benutzt die elektronische Kirche sämtliche psychologischen Marketing- und Public Relations-Methoden. Neben Heilsversprechungen, der Bereinigung von Sünden, Gesundheit, Wohlstand und Freude bietet die elektronische Kirche auch ,kostenlose' Geschenke, wie religiöse Bücher, Broschüren, Platten, Jesus-Anstecknadeln, Puppen und andere Geschenke gegen die Entsendung einer Spende. Auf diese Weise erhalten die Fernsehprediger Namen und Adressen ihrer Zuschauer, die sie für ihre computerisierte Adressverwaltung und ihren Briefversand verwen-

35 Jim Montgomery, The Electric Church. In: The Wall Street Journal (19. Mai 1978), 1, 29.

36 Vgl. TIME, Back to that Oldtime Religion. In: TIME (26. Dezember 1977), 52-58.

37 Für eine Definition von „popular culture“ siehe Robert K. Merton, Social Theory and Social Structure. Glencoe, Illinois: Free Press 1957, 441: Merton setzt „popular culture" gleich mit "mass beliefs" und "public opinion"; ebenso Harold L. Nieburg, Public Opinion, Tracking and Targeting. New York: Praeger Publishers 1984, 34-38. 
den. Die Zuschauer erhalten regelmäßig Post von ihrem Fernsehprediger, der ihnen schriftlich Segen spendet und um neue Spendengelder bittet. So wird eine Nähe zwischen dem Televangelisten und dem Publikum suggeriert. Während der Sendung werden gebührenfreie Telefonnummern eingeblendet für die Zuschauer, die ein Bonus-Geschenk erhalten oder eine Spende entrichten wollen. Die Telefonnummern, unter denen man spirituelle oder allägliche Lebensberatung von Mitarbeitern der elektronischen Kirche erhalten kann, sind allerdings nicht gebührenfrei. ${ }^{38}$

Neben dem missionarischen Eifer, viele Menschen zu evangelisieren, haben die Fernsehprediger der elektronischen Kirche auch soziale und politische Ziele. Nach einer gemeinsamen Studie der Annenberg School of Communications der Universität Pennsylvania und dem Meinungsforschungsinstitut Gallup von 1984 werden in den Programmen der elektronischen Kirche selten theologische Themen angesprochen. Stattdessen überwiegen soziale, moralische und politische Themen. ${ }^{39}$

Jeffrey K. Hadden und Anson Shupe betonen, daß die Anpassung der religiösen Botschaft an das Medium Fernsehen eine Veränderung dieser Botschaft mit sich bringt.

Jeffrey K. Hadden und Charles E. Swann sehen in der elektronischen Kirche mehr als eine religiöse Erscheinung. Sie bezeichnen sie als ein soziales und kulturelles Phänomen mit der Kraft einer neuen Gegenkultur, die im Gegensatz zum "counterculture movement“ der 60er Jahre keine links-liberalen, sondern rechtskonservative und zivilreligiöse Werte vertritt. Als Teil einer kulturellen Revolution bringt sie alte, wiederbelebte Verhaltensmuster und Wertvorstellungen mit sich. Mit ihrem Buch „Prime Time Preachers - The Rising Power of Televangelism" prägten Hadden und Swann für dieses gesellschaftliche und für Amerika spezifische Phänomen den hybriden Begriff „televangelism“.40

Kritiker dieses Phänomens - das sind Vertreter der organisierten und etablierten Hauptkirchen Amerikas, liberale Journalisten, aber auch konservative Vertreter aus den eigenen Reihen - schließen sich der Ansicht Martin E. Martys an. Er warnt vor dem potentiellen negativen Einfluß der elektronischen Kirche und ihrer „unsichtbaren Religion" für die traditionelle Kirchengemeinde. ${ }^{41}$

38 Vgl. Franklin B. Krohn, The Sixty-Minute Commercial: Marketing Salvation. In: Humanist (November/Dezember 1980), 28-29.

$39 \mathrm{Vgl}$. George Gerbner/Larry Gross/Stewart Hoover/Michael Morgan/Nancy Signorielli/Harry E. Cotugno/Robert Wuthnow, Religion and Television. A Research Report by The Annenberg School of Communications, University of Pennsylvania and the Gallup Organization, Inc., 2 Bände, Pennsylvania, Philadelphia: The Annenberg School of Communications, Univ. of Pennsylvania, April 1984, dort Bd.1, 22-58.

40 Vgl. Jeffrey K. Hadden/Charles E. Swann, Prime Time Preachers (Anm. 7), 3, 85.

41 Vgl. Martin E. Marty, „The Invisible Religion, "Presbyterian Survey, May 1979, 13 zit. n. Stewart M. Hoover, The Electronic Giant (Anm. 28), 117. 


\section{Der Erfolg der elektronischen Kirche}

Nach Angaben der Organisation der evangelikalen Rundfunkbetreiber NRB gab es 19871.370 religiöse Radiosender, 221 religiöse Fernsehsender und 4 große christliche Rundfunkanstalten, deren tägliches 24-stündiges Programm über Satelliten national ausgestrahlt wurde. Zusammen erreichten die Fernsehevangelisten der elektronischen Kirche in den 80er Jahren wöchentlich zwischen 13 und 20 Mio. Haushalte. ${ }^{42}$ Aufgrund ihrer marktbeherrschenden Stellung innerhalb des religiösen Rundfunks sprechen einige Beobachter von einem Monopol der elektronischen Kirche.

In den 50er Jahren waren Fernsehprediger der elektronischen Kirche noch auf periphere und kleinere Marktsegmente im lokalen Bereich beschränkt, während die organisierten Kirchen ihre Programme über ABC, NBC und CBS ausstrahlen konnten. In den 60 er und 70er Jahren hatten die unabhängigen lokalen Fernsehstationen sowie die lokalen „affiliates“ der nationalen Rundfunkanstalten die Rentabilität des Verkaufs von Sendeplätzen für unabhängig produzierte religiöse Programme der evangelikalen Prediger erkannt, so daß 1977 die evangelikalen Fernsehprediger bereits $92 \%$ der gesamten religiösen Sendezeiten einnahmen, während nur noch $8 \%$ der Sendezeiten kostenlos vergeben wurden. ${ }^{43}$

Die elektronische Kirche erlebte ihren größsten Aufschwung Anfang bis Mitte der 70er Jahre. Dagegen nahm die Zahl der kostenlos gesendeten „network“Programme, d. h. vorwiegend Produktionen der Mainline-Protestanten, Katholiken und Juden, drastisch ab.

In den 80 er Jahren waren die Mehrzahl (83,3\%) religiöser Sendungen syndikalisierte Programme, d. h. national verbreitete und ausgestrahlte Programme. Davon waren die meisten Produktionen von unabhängigen evangelikalen Gruppen.

Die Fernsehprediger der elektronischen Kirche erlangten zunehmend eine größere finanzielle Unabhängigkeit von den Rundfunkanstalten und Fernsehstationen. Dafür wuchs ihre finanzielle Abhängigkeit von den Zuschauern, mit deren Spenden sie Sendezeiten kauften. Einige Televangelisten versuchten, aus dem sog. „religious ghetto“ - den für religiöse Programme üblichen sonntäglichen Sendeblöcken, vor allem Sonntag morgens - auszubrechen und Sendezeiten an Wochentagen mit höheren Einschaltquoten zu erlangen. Dies ist vor allen Dingen solchen prominenten Televangelisten gelungen, die eigene Fernsehstationen oder Kabelsender besitzen oder solchen, die statt des traditionellen Gottesdienstes unterhaltende Talk-Shows moderieren. ${ }^{44}$

Um größere Einschaltquoten zu erlangen, gestalteten die Fernsehprediger der elektronischen Kirche im Laufe der Jahre publikumsattraktivere Sendungen. Statt herkömmlicher Gottesdienstübertragungen rückten unterhaltendere Sende-

42 Vgl. Jeffrey K. Hadden/Anson D. Shupe (eds.), Televangelism (Anm. 8), 86.

43 Vgl. Peter G. Horsfield, Religious Television. The American Experience (Anm. 30), 9f, 88-94.

44 Vgl. Peter G. Horsfield, Religious Television. The American Experience (Anm. 30), 95-99. 
formen, wie religiöse Talk-Shows, in den Vordergrund. Ihre Sendungen wurden häufiger in Studios mit Live-Publikum produziert und es traten Chöre und Sänger auf. Gebete wurden immer seltener gesprochen, stattdessen erlangten Spendenaufrufe eine größere Bedeutung. Über neue technische Übertragungsmöglichkeiten - Kabel und Satelliten - erreichten die Televangelisten ein erweitertes Publikum. Einige Fernsehprediger gründeten eigene Kabelsender, wie Pat Robertsons „CBN Cable Network“, Jim Bakkers „PTL Network“ oder Jerry Falwells „Liberty Broadcasting Network" (LBN).

Im folgenden sollen die drei Einflußfaktoren dargestellt werden, die wesentlich zum Erfolg und zu dieser einzigartigen Stellung der elektronischen Kirche innerhalb des religiösen Fernsehens in Amerika beigetragen haben: Der Einfluß der "Federal Communications Commission" (FCC), der Einfluß des amerikanischen kommerziellen Fernsehens und der Einfluß der religiös-konservativen Fernsehprediger selbst.

\subsection{Der Einfluß der "Federal Communications Commission" (FCC)}

Die „Federal Communications Commission“ (FCC) schafft als ausführendes Organ der amerikanischen Regierung den rechtlichen Rahmen, innerhalb dessen eine Interaktion zwischen Religion und Fernsehen überhaupt erst möglich ist. Kritiker behaupten, daß die FCC durch ihre Entscheidungen zum Aufstieg der elektronischen Kirche beigetragen habe. Dabei habe die dem Kongress unterstellte Aufsichtsbehörde die „establishment"-Klausel des ersten Zusatzartikels der Verfassung nicht beachtet, die die Errichtung, Unterstützung oder Förderung einer Religion untersagt..$^{45}$

Die Medienjuristin Linda Jo Lacey weist in ihrem ausführlichen Artikel auf unausgewogene Entscheidungen der FCC zugunsten der elektronischen Kirche hin. ${ }^{46}$

Zum Beispiel wirft Lacey der FCC eine zu milde Handhabung bezüglich der Geldbeschaffungsmethoden religiöser Prediger über den Äther vor. Weder die Werberegeln, die für das kommerzielle Fernsehen gelten, noch diejenigen, die für das öffentliche Fernsehen gelten, können für religiöse Programme angewandt werden. Zwar besteht für öffentliche, nicht-kommerzielle Sender ein striktes Verbot, während ihrer Sendungen für bestimmte Produkte oder Personengruppen Gelder zu erbitten, doch die FCC hat es versäumt, diese Regulienung bei religiösen Gruppen oder Organisationen durchzusetzen. Dadurch können diese Gruppen ungehindert Bibeln, Platten und anderen Produkte über den Äther verkaufen und neue Kunden anwerben.

Diese und andere FCC-Regelungen machten es für Fernsehsender lukrativer, ihre Sendezeiten an evangelikale und fundamentalistische Gruppen zu verkaufen, die bereit waren dafür zu bezahlen, als sie kostenlos an die Hauptkirchen zu vergeben.

45 Vgl. Linda Jo Lacey, The Electric Church: An FCC-“Established“ Institution? (Anm. 21), $235-236$.

46 Ebd. 
Zusammenfassend ist festzustellen, daß die „Federal Communications Commission" in der Vergangenheit meistens eine abneigende Haltung gegenüber der aktiven Einflußnahme auf religiöse Sendungen, insbesondere der elektronischen Kirche, gezeigt hat. Die FCC berief sich - wie auch die Fernsehprediger der elektronischen Kirche - immer wieder auf ihre im "Communications Act" festgelegte, neutrale Haltung und die im ersten Zusatzartikel der Verfassung garantierte freie Religionsausübung der Fernsehprediger, ihr Recht auf Rede- und Meinungsfreiheit und ihren Schutz vor einer Regierungskontrolle. Dadurch ermöglichte die Aufsichtsbehörde der elektronischen Kirche sowohl den Zugang zum öffentlichen, nichtkommerziellen als auch zum kommerziellen Fernsehen in Amerika. Trotz allem behandelte die FCC die Fernsehkirchen der konservativen Protestanten wie gemeinnützige Organisationen und wandte nicht die für kommerzielle Fernsehsender geltenden Regulierungen an. Sie ermöglichte den Televangelisten nicht nur den uneingeschränkten Kauf von Sendezeiten, sondern zeigte sich auch in ihren Entscheidungen bezüglich kontroverser und politischer Inhalte in religiösen Programmen ambivalent. Sie entzog sich der Verantwortung für die Inhalte, indem sie den Fernsehsendern die Aufgabe der Objektivität und Ausgewogenheit überließ.

\subsection{Der Einfluß des amerikanischen kommerziellen Fernsebens als Industrie und Institution}

Der Erfolg der Fernsehprediger beruht zu einem wesentlichen Teil auf dem wirtschaftlichen und sozialen Einfluß des Mediums Fernsehen in Amerika, der zweiten institutionellen Komponente des "televangelism" neben der Institution Religion im allgemeinen und der Institution der Erweckungsbewegungen im speziellen.

Die kommerzielle amerikanische Fernsehindustrie unterstützte durch ihre begünstigenden Richtlinien die evangelikalen Fernsehprediger, die zwar eine religiöse Minderheit darstellten, aber dafür zahlungsfähig und wettbewerbsorientiert waren. Außerdem herrschte zwischen dem amerikanischen Fernsehen und den Televangelisten eine Übereinstimmung bezüglich funktionaler Interessen:

Die Marktorientierung des kommerziellen amerikanischen Fernsehens bedingt eine starke Abhängigkeit des Fernsehens von der Werbeindustrie, deren ökonomische Interessen im Konfliktfall größere Beachtung finden als die innovativen oder kreativen Vorschläge der Fernsehproduzenten.

Die elektronische Kirche stellt eine Mischform zwischen dem öffentlichen Bildungsfernsehen („public television“) und dem kommerziellen Fernsehen dar: Die professionellen Televangelisten sind Teil der amerikanischen kommerziellen Fernsehindustrie, denn sie verkaufen ein Produkt: „Commercial television sells soap and hamburgers, while religious television sells redemption and salvation. ${ }^{47}$

Doch die Fernsehprediger werden nicht von Werbesponsoren finanziert, sondern von ihrem Publikum. Daher gelten für sie die FCC-Regelungen, die auch für religiöse Organisationen angewandt werden, und nicht die, die auch für kommerzielle Rundfunkbetreiber gelten. Um das sie finanzierende Publikum an sich zu

47 Razelle Frankl, Televangelism (Anm. 1), 9. 
binden, setzen die religiös-konservativen Fernsehprediger die gleichen Marketingund psychologischen Methoden ein wie das kommerzielle amerikanische Fernsehen einerseits und wie ihre Vorläufer, die Erweckungsprediger des 19. Jahrhunderts, andererseits. Die Erweckungsprediger unterwarfen sich dem äußeren Rationalisierungsprozeß, indem sie ihre Arbeitsweisen und ihre Organisations- und Rollenstrukturen standardisierten. Auch die kommerzielle Fernsehindustrie beugt sich dem wirtschaftlichen Druck, indem sie ihre Programmformate und damit ihre Arbeitsprozesse zu festgelegten Routinevorgängen macht. Kontinuität und Konsonanz durch standardisierte Programmuster, geregelte Sendezeiten und -längen und Werbeunterbrechungen im 15-minütigen Rhythmus erleichtern den Rundfunkanstalten den Verkauf ihrer Programme an ihre "affiliates“ oder andere unabhängige Sender.

Neben der traditionellen Messeübertragung verwenden die etablierten Fernsehprediger der elektronischen Kirche von den sechs Grundformaten des amerikanischen kommerziellen Fernsehens - episodische Serien, Nachrichten, Sport, Talk-Shows, Unterhaltungssendungen und Spiel-Shows - alle, außer Sportsendungen und Spiel-Shows. Ihre Sendungen bestehen ebenfalls aus professionell produzierten Einzelsegmenten, wodurch sie gegebenenfalls leicht zu kürzen sind. Bei der herkömmlichen Übertragung des Gottesdienstes aus einer Kirche oder während einer Zeltmission basiert der Inhalt dieser Einzelsegmente stark auf dem Einfluß der Erweckungspredigten: Neben musikalischen Einlagen kommt in der Sendung eine Predigt und eine religiöse Botschaft des charismatischen Fernsehpredigers vor, der eine dominante Rolle innerhalb der Sendung einnimmt. Ein weiteres Segment wird von einem Interview mit einer prominenten christlichkonservativen Persönlichkeit bestimmt. Segmente mit Geschenkangeboten, Spendenaufrufen und dem Angebot der Telefonseelsorge durch Mitarbeiter der Sendung durchbrechen die anderen Programmelemente.

Bei den religiösen „Infotainment“-Sendungen, deren Wohnzimmer-Kulisse den säkularen Talk-Shows des kommerziellen Fernsehens nachempfunden ist, bestehen die Segmente aus Interviews mit Gästen, magazinartigen Beiträgen über politische und ,human-interest"-Themen wie Gesundheit, Ernährung, Familienangelegenheiten usw., oder unterhaltenden Show-Einlagen und einem Segment, bei dem der Fernsehprediger seine Zuschauer zur Bekehrung und zum Spenden auffordert. Zwischen den beiden Extremformen - dem traditionellen, durch die Erweckungspredigten geprägten Gottesdienst einerseits und der durch säkulare Fernsehnormen geprägten Magazinsendung andererseits - gibt es verschiedene abgestufte Mischformen.

Gemeinsam ist allen die charismatische Autorität des Televangelisten, der bemüht ist, eine Vertrautheit und Intimität zu seinen Zuschauern aufzubauen. Dies gelingt ihm einerseits mit den produktionellen Mitteln des Fernsehens, d. h. durch eine subjektive Kameraführung (Nahaufnahmen des Fernsehpredigers), durch den vertrauten und freundschaftlichen Umgang mit den Mitwirkenden der Sendung (oftmals Familienmitglieder) und durch das Einbeziehen des Live-Publikums. Andererseits nähert sich die elektronische Kirche ihrem Publikum über die Programminhalte. Den gleichen ökonomischen Zwängen der freien Marktwirtschaft unterworfen, orientiert sich die elektronische Kirche an den Programmstrukturen, -inhalten und Produktionsweisen der kommerziellen amerikanischen Fern- 
sehindustrie. Die Televangelisten verfügen über die gleichen technischen Möglichkeiten der Fernsehproduktion wie kommerzielle Rundfunkveranstalter. Durch die Selektion von Inhalten und durch die Betonung, Aufmachung und Häufigkeit der Behandlung von bestimmten Themen (Agenda-Setting-Funktion) konstituiert die Fernsehkirche als Institution eine eigene soziale Realität.

Die elektronische Kirche ist eine Synthese zweier Institutionen, die ihrerseits ein eigenes gesellschaftliches Werte- und Normensystem, eigene Moralvorstellungen und stereotype Rollenverteilungen propagieren: Religion und Fernsehen sind beide Mythenproduzenten, deren Interpretation des Lebens eine eigene soziale Realität darstellt. Als kontinuierlicher Bestandteil der Alltagserfahrung tragen beide Institutionen zur Sozialisation und Kultivation von Realitätsvorstellungen der Gesellschaft bei. Problematisch erscheint die Tatsache, daß die durch die elektronische Kirche vermittelte soziale Realität eher der Realitätsdefinition des Fernsehens als der der christlichen Religion entspricht. ${ }^{48}$ William F. Fore stellt die durch das Fernsehen vermittelten „Mythen“ den christlichen Werten gegenüber: Die im Fernsehen vermittelte sozialdarwinistiche Vorstellung vom Überleben des Stärksten (impliziert Amerika als Weltmacht), die Vorstellung, daß der uneingeschränkte Erwerb von Konsumgütern Glück bedeutet und der Glaube, daß Reichtum, Eigentum, Macht und Fortschritt positive Ziele und wichtiger als soziales Engagement sind, stehen im Widerspruch zu den christlichen Vorstellungen, daß Geiz, Stolz und Unmäßigkeit Sünde sind und Nächstenliebe im Vordergrund stehen sollte. ${ }^{49}$

Für die evangelikalen und fundamentalistischen Fernsehprediger ist die Anpassung ihrer religiösen Botschaft an die ökonomischen und ideologischen Bedingungen des Fernsehens keineswegs paradox, denn ihre konservativ-religiöse Tradition befürwortet das kapitalistische System der freien Marktwirtschaft und den Einsatz aller technologischen Mittel für den Fortschritt. Wie ihre Vorläufer, die Erweckungsprediger, sind die Fernsehprediger selbst prominente, im Wohlstand lebende Persönlichkeiten, die sich damit rechtfertigen, daß Gott jene mit Erfolg und Reichtum belohnt, die sich seiner widmen. Ebenso in der evangelikalen Tradition verankert ist die Tendenz zur Dramatisierung, Sensationalisierung und Vereinfachung der religiösen Botschaft, die ein utilitaristisches Denken fördert.

Durch den direkten wirtschaftlichen Einfluß der amerikanischen Fernsehindustrie und den indirekten sozialen Einfluß der Institution Fernsehen erhielt die elektronische Kirche eine dominierende Position innerhalb des religiösen Fernsehens in Amerika. Auf diese Weise formte das Fernsehen die öffentliche Meinung, die den evangelikalen Fernsehpredigern mehr Einfluß zuschrieb als tatsächlich vorhanden.

\subsection{Der Einfluß der religiös-konservativen Fernsehprediger}

Der Erfolg der elektronischen Kirche wurde nicht nur durch äußere Faktoren bestimmt. Die religiös-konservativen Televangelisten trugen selbst wesentlich

48 Vgl. George Gerbner et al., Religion and Television (Anm. 39), Band 1, 12-14; Band 2, 47-58.

49 William F. Fore, Mass Media's Mythic World: At Odds with Christian Values. In: The Christian Century 94 (19. Januar 1977), 32-38. 
dazu bei, indem sie die Art der Finanzierung, das technologische Niveau, die Theologie und Ethik und die Inhalte der elektronischen Kirche bestimmten, wodurch diese sich vom religiösen Fernsehen der etablierten Großkirchen unterscheidet.

Die Televangelisten hatten im Gegensatz zu den Programmachern der Hauptkirchen eine relative wirtschaftliche Unabhängigkeit von der Fernsehindustrie erlangt. Gleichzeitig war ihre wirtschaftliche Abhängigkeit von ihren Zuschauern gewachsen.

Aus dieser Position heraus entwickelte sich eine "theology of technology" der evangelikalen und fundamentalistischen Fernsehpastoren. Damit ist eine teleologische, utilitaristische ethische Haltung gemeint, wonach alle Mittel einer zweckgerichteten Handlung gerechtfertigt werden, solange ihr eine berechtigte Motivation zugrunde liegt.

Die evangelikalen und fundamentalistischen Fernsehprediger waren schon immer offen für den technologischen Fortschritt. Quentin J. Schultze spricht vom „Mythos der elektronischen Kirche“, der begründet ist im technologischen Optimismus der Evangelikalen des 20. Jahrhunderts und ihrem Glauben an den christlichen Fortschritt durch den Einsatz modernster elektronischer Technologien. ${ }^{50}$ Sie beherrschten nicht nur alle Methoden der Telekommunikation, sondern waren auch mit der Computertechnologie und den professionellen Werbe- und Marketing-Methoden vertraut.

Die Pastoren der elektronischen Kirche kannten ihr Zielpublikum: Anhänger des evangelikalen, fundamentalistischen oder charismatischen Christentums, die politisch konservativ orientiert waren und eine Bereitschaft mitbrachten, sich in religiösen Angelegenheiten leiten und unterrichten zu lassen. Lm den verschiedenen psychologischen Ängsten und Bedürfnissen des Publikums - eine zunehmende "Verlassenheitserfahrung" aufgrund mangelnder Betreuung durch die herkömmlichen religiösen, politischen und sozialen Institutionen, ihre Angst vor einem moralischen Verfall Amerikas und ihr persönliches Bedürfnis nach Geborgenheit - entgegenzukommen, wandten sich die Fernsehprediger thematisch und methodisch an verschiedene Segmente dieses Zielpublikums.

„Während die Hauptkirchen die gute Nachricht von einem liebenden und vergebenden Gott predigen, der ewiges Leben gewährt, hat die elektronische Kirche bemerkt, daß man in unserer Zeit konkreter sein muß und auch unmittelbare Befriedigung versprechen muß." ${ }^{51}$

Einige evangelikale Fernsehprediger (z. B. Billy Graham, Rex Humbard, Oral Roberts, Jim Bakker, Robert Schuller und Jimmy Swaggart) boten ihren Zuschauern eine Religion, die so unterhaltsam, populär und inspirierend war wie die der Erweckungsprediger. Die populär inspirierende Religion machte von zivilreligiösen Symbolen Gebrauch - Patriotismus wurde als heilige Pflicht dargestellt.

50 Vgl. Quentin J. Schultze, The Mythos of the Electronic Church, in: Critical Studies in Mass Communication 4/3 (September 1987), 245-261.

51 Hans Schwarz, Die elektronische Kirche als Ausdruck amerikanischer Religiösität. In: Klaus M. Kodalle (Hg.): Gott und Politik in den USA, 1988, 124. 
Eine auf das Individuum bezogene Religion förderte, wie bei den Puritanern, eine Privatisierung des Glaubens. Im Mittelpunkt stand das persönliche Bekehrungserlebnis als Antriebskraft für individuelle und soziale Veränderungen, $\mathrm{d}$. h. nur der Glaube an Gott konnte zur Bewältigung persönlicher Probleme beitragen.

Ihr eigener wirtschaftlicher Erfolg und persönlicher Reichtum und Interviews mit bekehrten, prominenten und erfolgreichen Gästen aus der Wirtschaft oder Unterhaltungsbranche bestätigten, daß Gott einen starken Glauben mit Wohlstand, Glück, Freude und auch Ruhm belohnt. Während die Fernsehprediger selbst von der "Theologie des positiven Denkens" sprachen, beschrieben Kritiker diese Taktik als das „successful people syndrome“ bzw. eine „Wohlstands- und Erfolgstheologie". ${ }^{52}$

Während Fernsehprediger der Pfingstkirche oder charismatischen Bewegung eine höhere Emotionalität und Einbindung des Zuschauers boten - indem sie Heilungen und Wunder gegen Krankheit, Lebenskrisen oder beruflichen Mißerfolg versprachen - offerierten andere Televangelisten (z. B. Jerry Falwell, Pat Robertson und James Robinson) ihren Zuschauern einen sog. „kulturellen Fundamentalismus“, d. h. sie förderten die ethnozentristischen Gefühle vieler Amerikaner durch eine Kombination aus religiösem Fundamentalismus, politischem Konservatismus und einer patriotischen Zivilreligion.

Zwischen der populär inspirierenden Religion der einen und dem kulturellen Fundamentalismus der anderen Fernsehpastoren gab es verschiedene Mischformen. Allen gemeinsam war die vom Fernsehprediger beabsichtigte Personalisierung der Botschaft. Dies versuchte er durch direkte Spendenaufrufe und telefonische und postalische Zuschauerbetreuung, deren Ziel zum einen die Zuschauerbindung und damit die finanzielle Absicherung war, zum anderen die Evangelisierung, Missionierung und moralische Unterrichtung. Die Fernsehprediger setzten, wie ihre Vorläufer, die Erweckungsprediger des 19. Jahrhunderts, oder wie die modernen Werbetreibenden, Mittel der "mass persuasion" ein, d. h. Mittel der psychologischen Überzeugung unter dem Einfluß bestimmter Merkmale der Medienbotschaft. ${ }^{53} \mathrm{Um}$ eine effektive Medienwirkung zu erlangen,bedarf es neben der Bedürfnisbefriedigung des Publikums durch die vermittelte Botschaft auch der ergänzenden interpersonellen Kommunikation..$^{54}$

52 Vgl. William F. Fore, Beyond the Electronic Church. In: The Christian Century 98 (7.14. Januar 1981), 30.

53 Mittels des bewußten Einsatzes von Rhetorik oder anderen Reizen durch die Stimulusseite des Kommunikationsprozesses erzielen Werbetreibende und Prediger eine effektive und erfolgreiche Kommunikation, d. h. sie versuchen Einstellungs- und Verhaltensänderungen bei einem Massenpublikum zu bewirken. Für eine Analyse der persuasiven Sprache der Televangelisten vgl. Rosemarie Schmidt/Joseph F. Kess, Television Advertising and Televangelism. Discourse Analysis of persuasive language. Amsterdam/Philadelphia: John Benjamins Publishing Company 1986.

54 Vgl. Paul F. Lazarsfeld/Robert K. Merton, Mass Communication, Popular Taste, and Organized Social Action (1948), in: W. Schramm/D. F. Roberts (Hg.), The Process and Effects of Mass Communications. Revised Edition. Urbana: University of Illinois Press $1971,575-578,575 \mathrm{ff}$. 
Die evangelikalen Fernsehprediger gehörten zu den wenigen gesellschaftlichen Gruppen, die kostenintensive postalische und telefonische Kampagnen finanzieren konnten. ${ }^{55}$

Die Annenberg/Gallup-Studie von 1984 zeigte, daß die prominentesten Fernsehprediger am häufigsten und aggressivsten um Spenden baten. Das durchschnittlich erbetene Minimum war 31 Dollar und das durchschnittliche Maximum lag bei 600 Dollar. Als Spendengründe oder Anreize nannten die Televangelisten am häufigsten die Finanzierung ihrer Sendezeiten oder die Verbreitung des Evangeliums. Weitere Spendenappelle der charismatischen Televangelisten galten speziellen Projekten der Prediger (z. B. Bauvorhaben), finanziellen Krisen ihrer Fernsehkirchen, der Unterstützung wohltätiger Zwecke, moralischer Kampagnen (z. B. gegen Pornographie) und politischer Aktivitäten (z. B. für die rechtliche Durchsetzung des Schulgebets). Neben diesen direkten Spendenaufforderungen, boten die Televangelisten häufig auch Preise und Geschenke (z. B. Kassetten, Schallplatten und Bibeln, Schmuck) gegen Spenden. Mehrmals während der Sendung eingeblendete Telefonummern gaben den Zuschauern die Möglichkeit per Anruf ein Geschenk zu erwerben. Diese Methode der Spendenbeschaffung will eine Produktbindung herstellen, in diesem Fall an die elektronische Kirche.

Ähnlich wie beim mittelalterlichen Ablaßwesen boten ein Viertel der prominenten Fernsehprediger gegen eine Spende Heilungen körperlicher oder seelischer Gebrechen. Einige boten sogar eine materielleVergütung der Spende, d. h. sie versicherten auch die Heilung finanzieller Nöte ihrer Zuschauer oder die Erfüllung ihrer materiellen Wünsche. Das Prinzip dieser sofortigen Nutzen versprechenden „Gospel of Prosperity“ besteht darin, eine gegenseitige Abhängigkeit zwischen Fernsehpfarrer und Zuschauer herzustellen.

Manche evangelikalen Fernsehprediger offerierten ihren Spendern die Mitgliedschaft in einem Club ihrer elektronischen Kirche, wie Jim Bakkers „PTL-Club“ oder Pat Robertsons „700 Club“. Dadurch soll dem Zuschauer ein Gefühl der Zugehörigkeit, der Vertrautheit und der Gemeinschaft vermittelt werden, welches durch den computerisierten Postbeantwortungsservice und die telefonische Seelsorge gefördert wird.

55 Vgl. Peter G. Horsfield, Religious Television. The American Experience (Anm. 30), 84f; Robert Abelman, Religious Television Uses and Gratifications. In: JOBEM 31/3 (Sommer 1987), 2 
Während die Massenmediennutzung allgemein auch als „para-social interaction" bezeichnet wird ${ }^{56}$ kann man bei der elektronischen Kirche von einer utilitaristisch motivierten para-personalen Kommunikationsform sprechen. ${ }^{57}$ Jeffrey K. Hadden spricht vom Einfluß der "dritten Kommunikationsrevolution“, die die Entwicklung der elektronischen Kirche nachhaltig beeinflußt hat. Die Fernseh-Evangelisten, selbst „media managers" bzw. „media manipulators“, beeinflußen nun ihrerseits das religiöse, kulturelle und politische Leben Amerikas.

\section{Die politische und soziale Wirkung konservativer Protestanten und der Fernsehprediger}

Die elektronische Kirche bzw. das Phänomen des „televangelism“ läßt sich nur dann als gesellschaftliche und kulturell bedeutsame Erscheinung verstehen, wenn man das politische und soziale Wirken und den Einfluß der Fernsehprediger erkennt.

Der Einfluß der Evangelikalen und Fundamentalisten auf die amerikanische Innen- und Außenpolitik, Gesellschaftspolitik, Gesetzgebung und Bildungsinstitutionen war immer dann am größten, wenn die etablierten politischen und religiösen Institutionen nicht mehr auf die sich rasch verändernden sozio-ökonomischen Verhältnisse antworten konnten. Das politische Engagement konservativer Christen war zwar oftmals gegen die liberal-soziale Politik gerichtet, doch ihre Ziele orientierten sich weniger an einer bestimmten Parteipolitik als vielmehr an einer zivilreligiösen Interpretation der amerikanischen Verfassung.

Da sich die Anhänger der evangelikalen und fundamentalistischen Bewegung aus verschiedenen Denominationen rekrutieren, ist ihr Wahlverhalten nicht direkt bestimmbar. Umfragen ergaben, daß die evangelikalen, fundamentalistischen und charismatischen Christen zwar nicht einer bestimmten Partei zuzuordnen waren, doch im Vergleich zur Gesamtbevölkerung erwiesen sie sich als konservativer in ihren Einstellungen. Es war bezeichnend, daß Albert J. Menendez in seinem Buch über das Wahlverhalten religiöser Gruppen 1977 die Evangelikalen als „sleeping giant" charakterisierte. ${ }^{58}$ Der Wahlausgang im Jahre 1980 war für viele Amerikaner der Beweis, daß der schlafende, konservative Riese geweckt worden war.

56 Vgl. Donald Horton/R. Richard Wohl, Mass Communication and Para-Social Interaction. Observations on Intimacy at a Distance. In: „Psychiatry“, 19. Jg. 1956, 215-229 zit. n. Hans Mathias Kepplinger, Die Grenzen des Wirkungsbegriffs. In: Publizistik 27 (1982), 103: und zit. n. Jeffrey K. Hadden/Charles E. Swann, Prime Time Preachers (Anm. 7), 13, 65-67, 103f.: Die Para-soziale Interaktion der Massenmedien mit ihrem Publikum bedeutet, daß durch den ständigen Kontakt der Zuschauer mit gewissen Charakteren in Fernsehsendungen eine gewisse Familiarität und Intimität erzeugt wird. Der Zuschauer glaubt die Charaktere zu kennen und zu verstehen. Dieser Ansatz läßt sich mit Hilfe der Theorie der symbolischen Interaktion erklären.

57 Die elektronische Kirche geht über die parasoziale Interaktion hinaus, indem sie den direkten Kontakt mit den Zuschauern sucht, um eine größere Intimität zu erzeugen. Vgl. Hermann Boventer, Profanes Medium, Elektronische Kirche. Die religiöse Botschaft auf dem Bildschirm, in: ders. ( $\mathrm{Hg}$.), Ethik des Journalismus. Zur Philosophie der Medienkultur, Konstanz (Universitätsverlag) 1985, 215-377, 216.

58 Albert J. Menendez, Religion at the Polls. Philadelphia: Westminster Press 1977 zit. n. Jeffrey K. Hadden/Charles E. Swann, Prime Time Preachers (Anm. 7), 161. 
In den Augen der konservativen Christen Amerikas versagte in den 60er Jahren die liberale Politik und ihre neue Interpretation der Zivilreligion. Die Studentenunruhen, die Protestbewegungen gegen den Vietnam-Krieg und die Ghettoaufstände waren der Beweis, daß das politische System nicht in der Lage war, „peace, law and order" zu gewährleisten. Dieser Legitimitätsverlust politischer Institutionen wurde in den 70er Jahren durch die Immoralität der Nixon-Ära, die verfassungsrechtliche Unterstützung der säkularen Humanisten und die Legitimierung der Abtreibung durch den Supreme Court bestätigt.

Die steigende Unzufriedenheit mit dem politischen Establishment der Demokratischen und Republikanischen Partei äußerte sich einerseits durch die Fluktuation von Wechselwählern innerhalb des Parteiensystems, andererseits durch die politische Mobilisierung evangelikaler und fundamentalistischer Gruppen. Der Kernpunkt ihres "plan to save America" war die Rückkehr zur traditionellen Interpretation der Zivilreligion und deren fundamentaler moralischer Wertvorstellungen.

Zwar hatten Evangelikale und Fundamentalisten bis Mitte der 70er Jahre gepredigt, daß es dem christlichen Verständnis widerspräche, sich in die weltliche Politik einzumischen, doch angesichts des moralischen Wertewandels, den sie als „eklatanten Übelstand" empfanden, sahen sich einige fundamentalistische Fernsehevangelisten berufen, sich gleichzeitig um eine religiöse Renaissance zu bemühen und sich politisch zu engagieren.

Mit dem Ziel, religiöse wie nichtreligiöse Amerikaner in den Prozeß der moralischen Sanierung Amerikas einzubinden, gründeten evangelikale und fundamentalistische Christen - unter anderem Fernsehprediger wie Jerry Falwell - Ende der 70er Jahre diverse partei- und religionsunabhängige Interessensgruppen. Da die religiös-konservativen Fernsehprediger aus Vorsicht vor FCC-Maßnahmen in ihren Fernsehsendungen nur bedingt politische Inhalte ansprechen konnten, versuchten sie mittels dieser auf staats- und lokaler Ebene institutionalisierten Gruppen der sog. Neuen Christlichen Rechten („New Christian Right"; NCR) politisch Einfluß zu nehmen. Die NCR arbeitete eng mit der Neuen Rechten („New Right“; NR) zusammen. Letztere bezeichnet nicht nur die wiederauflebenden konservativen Kräfte in Amerika, sondern eine Gruppe von Kommunikations-, Werbe- und Marketingspezialisten, die außerhalb des institutionalisierten Parteiapparates agierte.

Gemeinsam ergriffen die NR und die NCR folgende Maßnahmen: Sie informierten die Öffentlichkeit über ihre Anliegen durch die Programme der elektronischen Kirche und anderer Medien und mobilisierten ,passive"Wähler, sich an Wahlen zu beteiligen. Sie wählten politische und parteipolitische Außenseiter, die sich im Kongreß für die rechtliche Durchsetzung ihrer Forderungen einsetzten. Dies zeigte sich bei den Wahlen zum Kongreß 1978 und 1984, bei denen die Niederlage einiger liberaler Senatoren und Kongreßabgeordneter herbeigeführt wurde und diese durch konservative Senatoren ersetzt wurden. ${ }^{59}$

59 Vgl. Allan J. Mayer, A Tide of Born-Again. In: Newsweek, 15. September 1980, 29, 32; James Mann/Sarah A. Peterson, Preachers in Politics: Decisive Force in '80? In: U.S. News and World Report (15. September 1980), 25. 
Die diversen Gruppen der Neuen Christlichen Rechten ${ }^{60}$ unterstützten kein einheitliches parteipolitisches Programm, sondern einzelne, moralisch besetzte soziale und politische Themen (,single issues"), wie beispielsweise die Abtreibungsfrage, die Durchsetzung des Schulgebetes an öffentlichen Schulen, den Kampf gegen die Lehre der Evolutionstheorie und den weltlichen Humanismus an öffentlichen Schulen, die Ablehnung der rechtlichen Gleichstellung von Frauen („Equal Rights Amendment") und Homosexuellen und die Zensur pornographischer und obszöner Schriften.

Politisch unterstützten sie Israel und das jüdische Volk, appellierten an die Wiederherstellung der militärischen Überlegenheit der USA - besonders gegenüber dem "gottlosen Kommunismus“.

Trotz ihrer verschiedenen Interessen fühlten sich die Gruppen der Neuen Christlichen Rechten verbunden in ihrem gemeinsamen Anliegen, den moralischen Verfall Amerikas aufzuhalten und die traditionelle Symbolik der Zivilreligon wiederzubeleben.

Dies erklärt die Wahl des zwar demokratischen, aber in erster Linie evangelikalen Jimmy Carter 1976 zum Präsidenten der Vereinigten Staaten. Zum ersten Mal setzte ein amerikanischer Präsidentschaftskandidat neben der zivilreligiösen Symbolik seine persönliche Religion ein, um die Kandidatur zu erlangen. Die Wahl Carters machte die amerikanischen Medien auf die große Zahl der Evangelikalen aufmerksam (Schätzungen schwanken zwischen 30 und 85 Mio.), was den Meinungsforscher George Gallup veranlaßte, das Jahr 1976 „The Year of the Evangelical“ zu nennen. Die Enttäuschung über Jimmy Carters Amtsperiode führte zu einer Veränderung des Wählerverhaltens zugunsten der Republikanischen Partei und zu Lasten der Demokratischen Partei. Bei den Wahlen 1980 wechselte die Mehrheit der Protestanten und der Katholiken von der Demokratischen zur Republikanischen Partei. Dieses Wahlergebnis führten die religiösen Gemeinschaften und die Medien auf die intensive Kooperation zwischen der NR und der NCR zurück.

Die Verbreitung politischer Ansichten von Predigern über den Rundfunk war kein neues, jedoch ein seltenes Phänomen in der Geschichte des religiösen Rundfunks. In den 80er Jahren äußerten Fernsehprediger der NCR zunehmend auch in ihren Programmen offen ihre konservativen Ansichten zu den moralisch relevanten Themen und riefen ihre Zuschauer auf, bei den Wahlen den republikanischen Präsidentschaftskandidaten Ronald Reagan zu unterstützen.

So zeigen die wenigen Inhaltsanalysen, die zu den Programmen der Televangelisten erstellt wurden, daß die Behandlung religiöser Themen Mitte der 80er Jahre merklich zurückging. Gleichzeitig räumten die konservativen Fernsehprediger sozialen und politischen Inhalten mehr Sendezeit ein. ${ }^{61} 1983$ machten religiöse

60 Zum Beispiel „Moral Majority, Inc.“, „Christian Voice“, ,the Conservative Caucus“, „the (Religious) Roundtable“, etc. Vgl. Jeffrey K. Hadden/Charles E. Swann, Prime Time Preachers (Anm. 7), 133-140; Perry Dean Young, God's Bullies. Native Reflections on Preachers and Politics. New York: Holt, Rinehart and Winston 1982, 95.

61 Vgl. George Gerbner et al. (...) (Anm. 39), Religion and Television, Band 1, 8f, 41-47; Robert Abelman/Kimberly Neuendorf, How Religious is Religious Television Programming? In: JOC 35/1 (1985), 98-110; Robert Abelman/Gary Pettey, How Political is Religious Television? In: JQ 65/2 (Sommer 1988), 313-319. 
Themen drei Viertel des gesamten Programmangebotes der prominentesten Televangelisten aus. Nur ein Viertel der Sendezeit wurde sozialen und politischen Themen gewidmet, wovon letztere nur knapp $4 \%$ ausmachten. 3 Jahre später bestand knapp $20 \%$ des Programmangebotes dieser Televangelisten aus politischen Themen.

In ihrer Öffentlichkeitsarbeit wurden die NCR und ihre konservativen Fernsehprediger indirekt durch die amerikanischen Massenmedien unterstützt. 1976 hatten die Evangelikalen erstmals die Aufmerksamkeit der Medien auf sich gelenkt. Neben religiösen Fachzeitschriften - wie"Christianity Today" und "Christian Century $^{\text {" }}$ - widmeten die großen nationalen und internationalen Zeitungen und Zeitschriften und die nationalen Fernsehanstalten ABC, NBC und CBS der erstarkenden evangelikalen Bewegung und ihrer elektronischen Kirche mehr Platz und Sendezeit.

Im Herbst 1980 behauptete die Neue Christliche Rechte, bereits 2-3 Mio. amerikanische "wiedergeborene" Christen in die Wählerliste eingetragen zu haben, und die Medien sprachen von dem ausschlaggebenden Einfluß dieser konservativen politisch-religiösen Bewegung auf die Wahl Reagans.

Im selben Jahr unterstützte Ronald Reagan erstmals öffentlich die Evangelikalen. Er kritisierte die "moralische Neutralität" der amerikanischen Regierung, des Obersten Gerichtshofes und anderer amerikanischer Institutionen und versprach, den Kreuzzug für eine moralische Erneuerung Amerikas mit anzuführen. Er versicherte, daß seine Administration zu den traditionellen moralischen Werten in der Politik zurückkehren werde. Seine Legitimierung der konservativen Christen zeigt, daß auch Reagan die "moral majority“ als die neue öffentliche Meinung erkannte. Sie zu mißachten, hätte ihm den Wahlsieg kosten können.

Schließlich gelang es der NCR und den konservativen Televangelisten, sich auch innerhalb des politischen Institutionensystems Gehör zu verschaffen, um ihre Forderungen durchzusetzen. Das Oberste Gericht änderte in einigen Fällen seine Rechtsprechung zur Abtreibung, Pornographie und zur Pressefreiheit zugunsten der NCR.

Es bestand also eine gegenseitige Zusammenarbeit und Abhängigkeit zwischen der NCR, ihren konservativen Fernsehpredigern und der Regierung, wobei diese Verbindung auch von kirchlichen und politischen Kreisen kritisiert wurde. ${ }^{62}$ Der damalige Vize-Präsident George Bush, eigentlich Mitglied der Episkopalkir-

62 Neben den liberalen protestantischen, katholischen und jüdischen Mainline-Kirchen wurde auch aus den eigenen evangelikalen Reihen Kritik gegen dieses neue politische Engagement geäußert, denn es entsprach nicht dem historischen Prinzip der Nichteinmischung religiöser Gruppen in die säkulare Politik. Bestehende links-liberale Bewegungen, wie "Common Cause“ und die "American Civil Liberties Union" (ACLU), mobilisierten sich und neue „anti-New Religious Right"-Organisationen, wie „People for the American Way“, „Americans for Common Sense" und „Moral Alternatives in Politics“, wurden gegründet. Vgl. Jeffrey K. Hadden/Charles E. Swann, Prime TimePreachers (Anm. 7), 143-148, 153. 
che, bezeichnete sich als „wiedergeborenen " Christen und kommunizierte mit den Fernsehevangelisten Billy Graham und Jerry Falwell, um ebenfalls das Vertrauen der Neuen Christlichen Rechten und ihrer Fernsehprediger zu gewinnen.

Neben Bush war 1986 der Televangelist Pat Robertson einer der aussichtsreichsten Präsidentschaftskandidatenbewerber. Indem Robertson seine elektronische Kirche und das dazugehörige Kommunikationsnetz einsetzte, mobilisierte er nicht nur seine evangelikalen Anhänger. Er fand Zuspruch bei vielen konservativen Wählern, die sich nach der moralischen Sanierung Amerikas sehnten, die Reagan versprochen, aber nicht vollbracht hatte. Für seine Wahlkampfkampagne 1988 standen Robertson über 11 Mio. Dollar Spenden zur Verfügung.

Es ist schwierig festzustellen, inwieweit die Fernsehprediger und die NCR in den 80er Jahren tatsächlich die Wähler und damit den Ausgang der Wahlen beeinflußt haben, denn ihr Wirken fand während einer Phase der allgemeinen politischen Rechtsorientierung in Amerika statt. Nichtsdestotrotz wurden sie sowohl von Reagan als auch von den Medien und der Öffentlichkeit als bedeutsame gesellschaftliche Gruppierung angesehen. Ihre politischen, sozialen und rechtlichen Vorschläge, die die konservativen Protestanten und die Televangelisten veröffentlichten, wurden von der Regierung akzeptiert und oftmals auch legitimiert. Die Kraft der religiös und politisch konservativen Protestanten liegt in ihrem Zugang zum Medium Fernsehen, der es ihnen ermöglicht, ihre loyalen Anhänger zu gesellschaftlichen Aktionen zu mobilisieren.

\section{Zuschauer der elektronischen Kirche}

Da die Zuschauer, die sich von den Televangelisten zu Spenden zur Finanzierung der elektronischen Kirche mobilisieren lassen, eine potentielle politische Kraft darstellen, sind sie für die Fernsehprediger der elektronischen Kirche von größerer Bedeutung als für Prediger religiöser Fernsehsendungen der MainlineKirchen.

Als 1980 die Medien über den potentiellen Einfluß der elektronischen Kirche auf die Wahlen berichteten, stieg das öffentliche Interesse an der politischen und sozialen Bedeutung des „televangelism". Kritiker behaupteten, die elektronische Kirche fördere eine Privatisierung der Religion, d. h. immer mehr Menschen würden das private religiöse Erlebnis der Fernsehkirche dem gemeinschaftlich erlebten Kirchenbesuch vorziehen. ${ }^{63}$ Einher ging die Frage nach den Einschaltquoten der Programme der Fernsehprediger. Bezüglich ihrer Schätzungen über das eigene Publikum lagen die Televangelisten in der Tradition der evangelikalen Erweckungsprediger, die die Zahl der bekehrten Seelen als Zeichen des Erfolges deuteten und daher zu Übertreibungen neigten: Ben Armstrong, Vorsitzender der "National Religious Broadcasters“ (NRB), eröffnete sein Buch "The Electric Church“ (1979) mit einer Schätzung des wöchentlichen Gesamtpublikums der elektronischen Kirche von 130 Mio. Zuschauern. Während Jim Bakker das Publikum seiner Sendung „PTL-Club“ auf 20 Mio. Zuschauer einschätzte, sprach Jerry Falwell 1980

63 Vgl. Hermann Boventer, Profanes Medium, Elektronische Kirche (Anm. 57); Robert Wuthnow, The Social Significance of Religious Television. In: Review of Religious Research 29/2 (Dezember 1987), 126-128. 
bei der nationalen republikanischen Wahlversammlung in Detroit vor Journalisten von 50 Mio. Zuschauern, die seine Sendung wöchentlich sahen. Kritiker der elektronischen Kirche - insbesondere Mitglieder des Nationalrats der Mainline-Protestanten NCC - behaupteten dagegen, daß insgesamt höchstens 10 Mio. Amerikaner wöchentlich die Programme der Televangelisten einschalteten. Im Verlauf dieser Kontroverse um die tatsächliche Größe des Publikums der elektronischen Kirche - auch "the great audience-size debate" genannt - wurden Anfang der 80er Jahre neue Studien zu den Einschaltquoten religiöser Programme veröffentlicht. Doch unterschiedliche Definitionen, Erhebungs- und Meßmethoden der verschiedenen Untersuchungen führten zu differierenden Ergebnissen, die sich nur schwer miteinander vergleichen lassen.

\subsection{Einschaltquoten}

Es gibt nur wenige Zahlen über das gesamte Publikum aller religiösen Fernsehsendungen, $d . h$. alle von den überregionalen Sendeanstalten produzierten, lokalen Sendungen und Kabelprogramme sowie „specials“ (z. B. Billy Grahams Sendungen) inbegriffen. Nach einigen Schätzungen sahen Ende der 70er/Anfang der 80er Jahre etwa 40 Mio. erwachsene Amerikaner wöchentlich mindestens eine Stunde religiöse Sendungen.

Die hier geschilderten Angaben beziehen sich im wesentlichen auf die Programme der elektronischen Kirche und andere "syndicated religious programs", d. h. national syndikalisierte und ausgestrahlte religiöse Programme denominationell unabhängiger Gruppen, die die Mehrheit des religiösen Programmangebotes in Amerika darstellen.

Die zwei privaten Zuschauerforschungsinstitute, A. C. Nielsen Company und Arbitron Company, die kontinuierlich die Hör- und Sehgewohnheiten des lokalen, regionalen und nationalen Radio- und Fernsehpublikums in den USA untersuchen, liefern auch regelmäßig Informationen über die national ausgestrahlten religiösen Programme.

Veröffentlichte Nielsen- und Arbitron-Zahlen über das durchschnittliche monatliche Gesamtpublikum syndikalisierter religiöser Programme zeigen einen deutlichen Trend: Das größte Wachstum erlebten die Sendungen der elektronischen Kirche zwischen 1970 und 1976. In dieser Zeit hatte sich gleichzeitig die Gesamtzahl der syndikalisierten religiösen Programme nahezu verdoppelt als auch das monatliche Gesamtpublikum für diese Sendungen von knapp 10 Mio. auf knapp 23 Mio. Zuschauer erhöht. Davon schauten über die Hälfte die syndikalisierten Programme der zehn populärsten Fernsehprediger. Während 1977 die Gesamtzahl der syndikalisierten religiösen Programme zurückfiel und sich das monatliche Gesamtpublikum verringerte, erreichte der Anteil der monatlichen Zuschauer der Sendungen der "top ten"-Fernsehprediger mit über 15 Mio. Zuschauern einen vorläufigen Höhepunkt. Bis 1981 fluktuierten diese Zahlen, um sich auf ein monatliches Gesamtpublikum von 21,7 Mio. Zuschauern für alle 64 national ausgestrahlten religiösen Programme einzupendeln. Davon schauten fast 14 Mio. Personen die Programme der 10 populärsten Fernsehprediger.

Diese Erkenntnisse stießen auf die Kritik der Televangelisten,die sich auf weitaus höhere Einschaltquoten beriefen. Dagegen kritisierten liberale Protestan- 
ten, daß die Daten viel zu hoch seien. Aufgrund dieser Auseinandersetzung beauftragte ein gemeinsamer Ausschuß von liberalen und konservativen Rundfunkbetreibern die Annenberg School of Communications der University of Pennsylvania und die Gallup Organization mit einer nationalen Studie über die Zuschauer religiöser Fernsehsendungen. ${ }^{64}$

Die beiden Forscherteams hatten nicht nur unterschiedliche Meßmethoden angewandt, sondern kamen auch zu unterschiedlichen Zuschauerschätzungen: Das Annenberg Team hatte zwei regionale Untersuchungen im Nordosten und Südosten Amerikas durchgeführt. Gegenstand der Analyse waren die wöchentlichen Fernsehtagebücher von 2.602 Fernsehzuschauern. Nach dieser Studie wurde das wöchentliche Publikum syndikalisierter religiöser Fernsehsendungen auf 13,3 Mio. Zuschauer geschätzt bzw. 6,2\% der Personen aller Fernsehhaushalte in den USA. Das Gallup-Team hatte im Mai 1983 eine nationale repräsentative Umfrage unter 2.003 Personen durchgeführt und kam zu dem Ergebnis, daß etwa jeder dritte Erwachsene (bzw. $32 \%$ der Befragten, das entspricht etwa 70 Mio. Zuschauern) in den letzten 30 Tagen und $18 \%$ (das entspricht etwa 40 Mio. Zuschauern) in den letzten 7 Tagen ein religiöses Fernsehprogramm gesehen hatten.

Da keine der beiden Studien verkabelte Haushalte berücksichtigten, beauftragte der Fernsehprediger Pat Robertson die A. C. Nielsen Company, eine Untersuchung für seinen christlichen Kabelsender CBN und die "top ten" der religiösen Programme der elektronischen Kirche durchzuführen. Sowohl diese Untersuchung, als auch eine Gallup-Umfrage von 1987 kamen zu dem Ergebnis, daß 39\% der Amerikaner in den letzten 30 Tagen und $25 \%$ in der vergangenen Woche ein religiöses Programm gesehen hatten. ${ }^{65}$

Peter G. Horsfield argumentiert, daß - ungeachtet aller meßmethodischen Differenzen - höchstens 10-15 Mio. Zuschauer wöchentlich regelmäßig die Sendungen der elektronischen Kirche sähen, wobei viele dieser „Seher“ mehrere syndikalisierte religiöse Programme schauten, was zu einer erhöhten Gesamtzahl der Zuschauer führe.

Die hier zitierten Zuschauerzahlen für alle syndikalisierten religiösen Programme und die der populärsten Televangelisten legen die Vermutung nahe, daß die elektronische Kirche 1977/78 - zumindest zahlenmäßig gesehen - ihren größten Einfluß hatte und nicht im Wahljahr 1980, als die Medien durch ihre Fokussierung das öffentliche Interesse an ihr weckten.

Die Debatte um die Einschaltquoten ließ oftmals demographische, soziale, kulturelle und religiöse Faktoren außer acht, ohne die sich die elektronische Kirche als soziales und politisches Phänomen nicht begreifen läßt.

64 Vgl. George Gerbner/Larry Gross/Stewart Hoover et al. (eds.), Religion and Television (Anm. 39).

65 Vgl. David W. Clark/Paul H. Virts, Religious Television Audiences: A New Development in Measuring Audience Size. Unveröffentlichter Vortrag vor der Jahresversammlung der "Society for the Scientific Study of Religion“, Savannah, Georgia, 25. Oktober 1985 zit. n. Jeffrey K. Hadden/Anson D. Shupe (eds.), Televangelism (Anm. 8), 154157. 


\subsection{Demographische, sozio-ökonomische und religiöse Merkmale}

Über die demographischen, sozio-ökonomischen und religiösen Eigenschaften des Publikums religiöser Fernsehsendungen herrscht in der Wissenschaft eine größere Übereinstimmung als bezüglich der Einschaltquoten. Die Annenberg/Gallup-Studie von 1984, die "Seher" (Personen, die in den vergangenen 30 Tagen ein religiöses Programm angeschaut hatten) religiöser Programme mit den „Nichtsehern" verglich, bestätigte die Erkenntnisse der meisten Studien über das religiöse Radio- und Fernsehpublikum, die seit Mitte der 50er Jahre erstellt worden waren:

„Die Zuschauer religiöser Programme sind im großen und ganzen auch die Gläubigen, die Kirchgänger, die Spender. Ihr Zuschauen ... scheint Ausdruck, Kultivierung und Bestätigung einer Reihe von religiösen Anschauungen zu sein und nicht ein Ersatz für diese. Das Profil dieses Publikums religiöser Sendungen scheint ... etwas älter, mit geringerer Ausbildung und Einkommen, konservativer und "fundamentalistischer" zu sein, und sie scheinen eher in ländlichen Gegenden und im Süden und Mittleren Westen zu leben als solche Personen, die keine religiösen Programme sehen." ${ }^{\prime 6}$

Obwohl festgestellt worden ist, daß beim nationalen Fernsehpublikum Sonntagsmorgens das Verhältnis zwischen Männern und Frauen ausgewogen ist, sind die Zuschauer religiöser Fernsehsendungen überwiegend weiblich. In der Annenberg/Gallup-Studie sahen $44 \%$ der befragten Männer und $56 \%$ der befragten Frauen religiöse Programme. Frauen fielen auch häufiger in die Kategorie der "Viel-Seher" (die mehr als 2 Stunden wöchentlich religiöse Programme sahen) als Männer.

Über die Hälfte der Zuschauer ist 50 Jahre alt und älter, wobei ältere Frauen häufiger religiöse Sendungen schauen als ältere Männer. Dabei ist die Anzahl der verwitweten und geschiedenen Frauen höher als bei den Personen, die keine religiösen Sendungen sehen. Nur etwa ein Fünftel aller in der Annenberg/GallupStudie befragten „Seher" waren jünger als 30 .

Personen mit geringeren Einkommen und geringerer Ausbildung sehen signifikant häufiger religiöse Sendungen als Personen in höheren Einkommensklassen mit einer besseren Ausbildung. Nur $10 \%$ der befragten "Seher" in der Annenberg/Gallup-Studie von 1984 verfügten über einen College-Abschluß, wohingegen über ein Drittel (38\%) nicht einmal die Highschool abgeschlossen hatte. Dagegen überwog bei den "Nichtsehern" die Zahl der Highschool-Absolventen (43\%) und etwa ein Fünftel (19\%) von ihnen hatte ein College-Diplom.

Es wurde deutlich, daß die meisten Zuschauer religiöser Fernsehsendungen ältere Menschen mit einer geringeren Ausbildung sind ( $52 \%$ hatten keinen Highschool-Abschluß). Die Gruppe der unter 30-jährigen mit College-Abschluiß (13\%) schaute diese Programme dagegen nur selten. Das Meinungsforschungsinstitut Gallup nennt soziale Isolation, die schlechtere physische und psychische Verfas-

66 Von der Verfasserin ins Deutsche übersetzt. Für Originàlzitat siehe George Gerbner et al., Religion and Television (Anm. 39), Band 2, 2-3. 
sung und die reduzierte Mobilität älterer Menschen als Gründe dafür, daß ältere Personen häufig religiöse Shows sehen.

Bei der Kategorisierung in "weiße" und „farbige“ Amerikaner zeigte sich, daß die Mehrzahl der "Seher“ (81\%) „weiß“ war und nur ein Fünftel (19\%) rassischen Minderheiten angehörte.

Obwohl die meisten religiösen Sendungen, vor allem die Sendungen der prominenten Televangelisten der elektronischen Kirche, landesweit und in fast allen Großstädten Amerikas ausgestrahlt wurden, ließen sich ihre Zuschauer geographisch fixieren. Zwar waren sie proportional gleichhäufig in Städten, Vorstädten und ländlichen Gebieten wohnhaft, doch im Vergleich zu „Nichtsehern" lebten verhältnismäßig mehr "Seher“ in ländlichen Gebieten (36\% zu 27\%). Regional betrachtet, konzentrierten sie sich auf den Süden und mittleren Westen Amerikas. Etwa zwei Drittel der Zuschauer religiöser Programme lebte in diesen vom Fundamentalismus geprägten Gegenden, die auch als Bibel-Gürtel („Bible Belt") bezeichnet werden. ${ }^{67}$ In den östlichen Staaten, wo 1980 etwa ein Fünftel (22,5\%) aller Amerikaner lebten, werden dagegen religiöse Programme seltener gesehen.

Die Zuschauer religiöser Sendungen unterschieden sich auch bezüglich ihrer Religiösität von Personen, die solche Programme nicht schauten. Fast drei Viertel der untersuchten "Seher" waren Protestanten (72\%), wobei sie meistens baptistischen Denominationen und den Methodisten angehörten. Knapp 20\% der Befragten waren Anhänger der Römisch-Katholischen Kirche. Im Vergleich waren bei den "Nichtsehern" 40 \% Katholiken und die Hälfte Protestanten. Allerdings sollte man hier beachten, daß die meisten religiösen Fernsehsendungen von protestantischen, insbesondere evangelikalen Gruppen produziert werden.

Drei Viertel der befragten Zuschauer waren Kirchenmitglieder (77\%) und fast ebensoviele antworteten auf die Frage „How important is religion in your own life?": „Very important“ (71\%), während nicht einmal die Hälfte der befragten "Nicht-Seher" Religion als sehr wichtig einstuften. Das Publikum religiöser Sendungen ging auch regelmäßiger in die Kirche als die Personen, die sich solche Programme nicht anschauten.

Im Vergleich zu den befragten „Nicht-Sehern“ waren die „Seher" häufiger Evangelikale bzw. Fundamentalisten, d. h. sie glaubten signifikant häufiger an die Irrtumsfreiheit der Bibel, die sie als verbalinspiriertes Wort Gottes ansahen, sie behaupteten mehr als doppelt so oft ein Erlebnis der "Wiedergeburt" gehabt zu haben als Personen, die keine religiösen Programme sahen ( $55 \% \mathrm{zu} 24 \%$ ) und zwei Drittel der Befragten hatten einen missionarischen Eifer, der sie dazu bewegte, andere Leute zu evangelisieren.

67 Vgl. Stephen W. Tweedie, Viewing the Bible Belt. In: Journal of Popular Culture 11/4 (1978), 865, 873. Tweedie definiert diese Region als „a broad zone stretching from Virginia to northern Florida in the East and from Dakotas to central Texas in the West." 


\subsection{Motivationen und Gratifikationen}

Die Rezipientenforschung bezüglich religiöser Fernsehsendungen befaßt sich mehr mit der Mediennutzung, d. h. den Einschaltquoten, und dem Einfluß latenter Dispositionen der Empfänger als mit den Zuschauerabsichten bzw. den ihnen zugrunde liegenden Bedürfnissen und Motiven, solche Sendungen zu sehen. Geringer noch ist die Anzahl der wissenschaftlichen Untersuchungen über die Wirkungen der Sendungen der Televangelisten. Die Televangelisten versuchen, ihre Zuschauer mittels Methoden der „Persuasion" psychologisch und ideologisch zu beeinflussen. Da die Massenkommunikation als parasoziale Interaktion die Änderungen von Meinungen, Einstellungen und Verhaltensweisen erschwert, so der amerikanische Soziologe Robert K. Merton, muß eine charismatische Fernsehautorität die Motivationen und Bedürfnisse der Zuschauer ansprechen, um eine effektive Wirkung zu erzielen. ${ }^{68}$

Der in den 40er Jahren entwickelte „Nutzens-und-Belohnungs-Ansatz“ (,uses and gratifications approach"), der statt der Absichten der Kommunikatoren die Bedürfnisse der Empfänger in den Mittelpunkt des Forschungsinteresses stellt, erlangte Ende der 60er/Anfang der 70er Jahre große Bedeutung in der Kommunikationsforschung.

Untersucht werden einerseits psychische (bzw. kognitive und affektive) und soziale (bzw. integrative und interaktive) Bedürfnisse der Medienrezipienten, anderseits der vom Publikum erwartete Nutzen (gratifications sought) und die tatsächlich erlangte Befriedigung dieser Bedürfnisse oder die Belohnung für die von Motivationen begründete Handlung (gratifications obtained). Schließlich wird die weiterführende Frage nach den Folgen gestellt, $d$. h. wie sich diese Erfahrungen der Rezipienten in ihrem Kommunikationsverhalten manifestieren. Bei diesem Forschungsansatz werden also die Leser, Hörer und Zuschauer nicht als homogenes, passives Massenpublikum betrachtet, sondern als aktive, zielorientierte Teilnehmer am Kommunikationsprozeß. schen:

Die „uses and gratifications“-Forschung unterscheidet im wesentlichen zwi-

- Dem Informationsbedürfnis, z. B. Befriedigung von Neugier und allgemeinem Interesse, Lernen und Weiterbildung.

- Dem Bedürfnis nach persönlicher Identität, z. B. Stärkung der persönlichen Werthaltungen, Suche nach Verhaltensmodellen, Identifikation mit anderen in den Medien.

- Dem Bedürfnis nach Integration und Interaktion, z. B. soziale Empathie, Identifikation mit anderen und Entwicklung eines Zugehörigkeitsgefühls, Kontaktaufbau zur Familie, Freunden und zur Gesellschaft.

- Dem Unterhaltungsbedürfnis, z. B. Wirklichkeitsflucht, Ablenkung von Problemen, Entspannung, kulturelle oder ästhetische Erbauung. ${ }^{()^{\circ}}$

68 Vgl. Robert K. Merton et al., Mass Persuasion. The Social Psychology of the War Bond Drive. New York/London: Harper \& Brothers Publ., 1946, $185 \mathrm{f}$.

$69 \mathrm{Vgl}$. Denis McQuail, Mass Communication Theory. An Introduction. London/Newbury Park/Beverly Hills/New Delhi: Sage Publications 21987. 73. 
Zwischen diesen Funktionen besteht oftmals eine gegenseitige Abhängigkeit.

Das Meinungsforschungsinstitut Gallup fragte die Zuschauer religiöser Programme nach ihren Motivationen, solche Sendungen zu sehen und faßte die Antworten in Kategorien zusammen. Jeder fünfte Befragte gab an, Gefallen an diesen Programmen zu finden, ohne dieses Vergnügen näher zu spezifizieren. Andere häufig genannte Gründe bezogen sich auf den religiösen Inhalt dieser Sendungen. Jeder siebte "Seher" erwähnte, daß religiöse Fernsehsendungen für ihn einen Ersatz für den Kirchenbesuch darstellten. Fast genauso viele nannten die "geistige Erbauung oder die Inspiration“, die ihnen diese Programme vermittelten. Die wenigsten Befragten - etwa jeder Fünfte - gaben Gründe an, die nicht den religiösen Inhalt der Sendungen betrafen, wie „Informationen/Lernen“ ( $8 \%$ ), „Das Programm war zufällig gerade an" (7\%) oder "Unterhaltung" (2\%).

In einem zweiten Schritt händigte das Gallup-Team den Zuschauern eine Liste mit 10 möglichen Gratifikationen aus, die sie von religiösen Fernsehsendungen erhalten könnten. Die Befragten wurden gebeten, all jene Aussagen anzukreuzen, die ihrer positiven Meinung über diese Programme entsprachen. Über die Hälfte der Befragten wählten „Die Predigt/das Gebet“ und jeweils ein Drittel nannte "die Musik“, „die geistige Erbauung“ und „das Gefühl der Nähe zu Gott". Die Zuschauer der elektronischen Kirche versprachen sich also in erster Linie einen religiösen Nutzen von diesen Programmen. Jeweils ein Viertel der "Seher" nannte außerdem „allgemeines Vergnügen“, „mehr über Weltereignisse erfahren“ und „Informationen über wichtige moralische oder soziale Themen erhalten". Seltener nannten die Befragten Gratifikationen, die vor allem von Kirchen befriedigt werden, wie „das Erlebnis des Gottesdienstes“, „sich als besserer oder stärkerer Mensch fühlen" oder "das Gefühl der Gemeinsamkeit oder Kameradschaft". Die Zuschauer religiöser Fernsehsendungen sind also von vornherein religiös motiviert, solche Sendungen zu sehen. ${ }^{70}$

Judith M. Buddenbaum stellte ebenfalls fest, daß die Motive, Bedürfnisse und Wertvorstellungen religiöser Zuschauer kirchenspezifisch und nicht medienbzw. fernsehspezifisch sind. ${ }^{71}$ Daraus leiten Kritiker der elektronischen Kirche das Argument ab, daß die Sendungen der Televangelisten eine institutionelle Bedrohung für die traditionellen Kirchen darstellen. ${ }^{72}$ Um herauszufinden, inwieweit sich

70 Damit bestätigt die Annenberg/Gallup-Studie Ergebnisse früherer Studien über die Motivationen der Zuschauer religiöser Fernsehsendungen und den Nutzen, den sie von diesen erwarten und erhalten. Für Dissertationen und andere wissenschaftliche Studien auf diesem Gebiet aus den sechziger und siebziger Jahren siehe Peter G. Horsfield, Religious Television. The American Experience (Anm. 30), 120-121; Robert Abelman, Motivations for Viewing "The 700 Club". In: JQ 65/1 (Sommer 1988), 114115.

71 Vgl. Judith M. Buddenbaum, Characteristics and Media-Related Needs of the Audience for Religious TV. In: JQ 58/2 (Sommer 1981), 266-272; vgl. auch Gary D. Gaddy/ David Pritchard, Is Religious Knowledge Gained From Broadcasts? In: JOC 35/1 (Winter 1985), 129.

72 Dieses Argument wurde insbesondere von dem Psychologen und Fernsehwissenschaftler Robert Liebert und von den liberalen Theologen Harvey Cox, Martin E. Marty und William F. Fore hervorgebracht. Vgl. Peter G. Horsfield, Religious Television. The American Experience (Anm. 30), 52-63: Jeffrey K. Hadden/Charles E. Swann, Prime Time Preachers (Anm. 7), 177f, 180-185. 
die Gratifikationen von Kirche und religiösen Sendungen überschneiden, fragte das Gallup-Team die "Seher" und "Vielseher" religiöser Sendungen und Personen, die mindestens einmal wöchentlich die Kirche besuchten, nach dem erwarteten Nutzen und den tatsächlich erhaltenen Gratifikationen, die sie von beiden Institutionen erhielten. Wie sich herausstellte, boten religiöse Fernsehsendungen viele Gratifikationen, die auch die Kirchen boten, nämlich Gebete, spirituelle Erbauung, und Musik. Gratifikationen, die nur selten vom Fernsehgottesdienst, aber vorwiegend von lokalen Kirchengemeinden befriedigt wurden, betrafen den Ritual- und Erlebnischarakter des kirchlichen Gottesdienstes, wie das Gefühl der Nähe zu Gott, der Eindruck der Gemeinsamkeit und Kameradschaft und das Gefühl, persönliche Stärke zu erlangen.

Die meisten Zuschauer der elektronischen Kirche waren sehr religiöse und engagierte Kirchenmitglieder. Sie waren der Meinung, daß die Evangelisierung, Missionierung und religiöse Unterrichtung primär die Aufgaben der lokalen Kirche und nicht des Fernsehgottesdienstes seien. ${ }^{73}$ Ein Viertel der in der Annenberg/ Gallup-Studie untersuchten Sendungen der elektronischen Kirche erwähnte die lokale Kirchengemeinde und 11,5\% forderten ihre Zuschauer auf, diese zu besuchen. Zwar folgten diesem Rat nur $7 \%$ der befragten "Seher“, aber noch geringer war die Anzahl derer, die sagten, daß sie aufgrund der Sendungen der Televangelisten ihre Kirchenaktivitäten (z. B. freiwillige Mitarbeit in der lokalen Kirchengemeinde oder Spenden an die Kirche) eingeschränkt hätten (3\%). Die meisten Zuschauer $(90 \%)$ gaben an, daß diese Sendungen keinen Einfluß auf ihre Kirchenaktivitäten hätten. Die Frage nach der Zufriedenheit mit der eigenen lokalen Kirche und deren Arbeit ergab, daß die Mehrheit der Zuschauer (71\%) mit dieser zufrieden war. Damit, so folgert die Annenberg/Gallup-Studie, scheint die Unzufriedenheit mit der lokalen Kirchengemeinde nicht der ausschlaggebende Beweggrund zu sein, sich den Televangelisten zu widmen.

Personen, die die elektronische Kirche als Ersatz für den Kirchenbesuch nutzten, waren vorwiegend ältere, alleinstehende Menschen, die weniger mobil waren und allgemein mehr fernsahen - sog. „Gewohnheits-Seher“ („habitual viewers"). Sie erwarteten von den Sendungen der Televangelisten die Befriedigung kirchenspezifischer als auch fernsehspezifischer Bedürfnisse, d. h. sie nutzten diese Programme als Informationsquelle, als Ersatz für fehlende Geselligkeit oder Partnerschaft, um Zeit zu füllen, als Entspannung und Erbauung und als Wirklichkeitsflucht. ${ }^{74}$ Eine andere Personengruppe, die religiöse Programme der Kirche vorzogen, waren Menschen, die mit ihrer örtlichen Kirche unzufrieden waren, d. $\mathbf{h}$. deren spezielle Bedürfnisse dort nicht befriedigt wurden.

73 Vgl. George Gerbner et al., Religion and Television (Anm. 39), Band 1, 4, 66; Band 2, 32; Gary D. Gaddy/David Pritchard, Is Religious Knowledge Gained From Broadcasts? In: JQ 64 (Winter 1986), 840-848: Gaddy und Pritchard kamen in ihrer Untersuchung zu dem Ergebnis, daß Zuschauer religiöser Sendungen ihr religiöses Wissen eher von traditionellen religiösen Medien (Bibel, Kirche, Schule) beziehen als von den christlichen Fernsehsendungen.

74 Vgl. Alan M. Rubin, Ritualized and Instrumental Television Viewing. In: JOC 34 (Sommer 1984), 69 
Die Motivationen der Zuschauer religiöser Sendungen unterscheiden sich also nicht nur von denen der Fernsehzuschauer allgemein. Auch innerhalb der Gruppe der religiösen Zuschauer gibt es Subgruppen, deren Motivationen unterschiedlich sein können. Allen gemeinsam scheint allerdings eine Unzuf riedenheit mit dem moralischen und religiösen Klima in Amerika zu sein.

Die Forscher der Annenberg/Gallup-Studie stellten fest, daß $84 \%$ der „Seher" unzufrieden waren über "the way moral standards have been changing in America“. Davon waren $50 \%$ "sehr unzufrieden“ (im Gegensatz zu den "NichtSehern“, von denen nur $31 \%$,sehr unzufrieden“ waren).

Eine Befragung zum Spendenverhalten der Zuschauer ergab, daß ein Viertel der befragten "Seher" schon einmal eine Spende an ein religiöses Fernsehprogramm entrichtet hatte, wovon allerdings nur die wenigsten (5\%) regelmäßig spendeten. Die meisten gaben an, ,hin und wieder" zu spenden oder nur aufgrund besonderer Spendenappelle der Televangelisten. Über die Hälfte der Befragten richteten ihre Spende nur an ein Programm, $10 \%$ gaben ihre Gelder 2 verschiedenen Programmen und nur $2 \%$ finanzierten 3 oder mehrere Programme. Nach der Höhe der Spende gefragt, gab jeweils etwa ein Viertel der Zuschauer an: „\$10 oder weniger" (7\%), ,zwischen $\$ 11$ und $\$ 29^{\prime \prime}(5 \%)$, ,zwischen $\$ 30$ und $\$ 74^{\prime \prime}(7 \%)$, , \$ 75 oder mehr" $(7 \%)^{75}$

Die regionale Untersuchung der Annenberg School of Communications zum Spendenverhalten zeigte zudem, daß die Spender nicht nur den oberen, sondern häufig auch den unteren Einkommensklassen angehörten. Stewart M. Hoover stellte in seinen Studien fest, daß die Spender der elektronischen Kirche nicht immer auch deren Programme sehen. Die Loyalität dieser Spender ergibt sich nicht aus den Sendungen der Televangelisten, sondern aus der Nutzung weitreichender Angebote ihrer parakirchlichen Unternehmen, die den Kontakt zu ihren Anhängern aufrechterhalten. ${ }^{76}$ Mehr als $30 \%$ der in der Annenberg/Gallup-Studie befragten Zuschauer berichteten, daß sie von einer solchen elektronischen Parakirche Spendenbriefe erhalten hätten und $11 \%$ gaben zu, ihrerseits brieflichen oder telefonischen Kontakt mit einer Fernsehkirche aufgenommen zu haben. So erhielten Jerry Falwell, Oral Roberts und Jimmy Swaggart durchschnittlich 10.000 bis 20.000 Briefe täglich, während Pat Robertson und Jim Bakker ebenso viele Zuschaueranrufe erhielten. ${ }^{77}$

75 Vgl. George Gerbner et al. (Anm. 39), Religion and Television, Band 2, 21, Tabelle 7 auf 22, 67-69, Tabellen 30,31: Die sozialen und religiösen Eigenschaften der Spender stimmen im wesentlichen mit den Eigenschaften der Zuschauer religiöser Sendungen überein, d. h. ältere, evangelikale, verheiratete Frauen, die regelmäßig die Kirche besuchen, spenden auch häufiger.

76 Stewart $M$. Hoover, The religious television audience: a matter of significance, or size?, in: Review of Religous Research 29/2 (Dezember 1987), 135-152, 148.

$77 \mathrm{Vgl}$. George Gerbner et al., Religion and Television, Band 2, 4, 22f.; Peter G. Horsfield, Religious Television. The American Experience (Anm. 30), 140f; Hans Norbert Janowski, Die kanalisierte Botschaft (Anm. 29), 73. 


\section{Zusammenfassung}

Die facettenreiche Erscheinung der elektronischen Kirche läßst sich nur durch die Aufarbeitung ihrer beiden institutionellen Komponenten - Religion und Fernsehen in den USA - begreifen. Erst die Verschmelzung dieser beiden wesentlichen Elemente der Alltagskultur in den USA brachte die elektronische Kirche bzw. das Phänomen des „televangelism“ hervor.

Seit Anfang des 17. Jahrhunderts prägt die Religion das private und öffentliche Leben der Amerikaner. Die von der spezifischen Religiösität der calvinistischen Puritaner und der Erweckungsprediger des 18. und 19. Jahrhunderts geformten Wertvorstellungen und Normen (Sendungsbewußtsein, Missionsgedanke, Aktivitätsprinzip, Arbeitsethos, Erfolgszwang, Individualismus etc.) und die sich daraus entwickelnde utilitaristische Ethik fanden Eingang in die öffentliche Zivilreligion Amerikas und bestimmen wesentlich die Ziele und Inhalte der elektronischen Kirche: Im Vordergrund steht die Evangelisierung und Bekehrung möglichst vieler Zuschauer, die die finanzielle Basis der elektronischen Kirche darstellen. Um dieses Ziel zu erreichen, predigen die Televangelisten entweder eine populär inspirierende Religion oder einen kulturellen Fundamentalismus mit zivilreligiösem Unterton. In beiden Fällen ist die Botschaft stark personalisiert, um ein Gefühl der Intimität zwischen Fernsehpastor und Zuschauergemeinde herzustellen. Hierin besteht eines der wesentlichsten Erfolgskriterien dieser religiös-konservativen Protestanten seit den 70er Jahren in Amerika. Im Gegensatz zu den etablierten Großkirchen, erkannten sie die Bedürfnisse ihrer Zuschauer: Die Unzufriedenheit vieler Amerikaner mit dem moralischen Klima und mit den politischen und sozialen Institutionen in ihrem Land und ihr gleichzeitiges Verlangen nach einfachen Erklärungsmustern, nach einer durch Autoritätsstrukturen gesicherten Stabilität und nach einem emotionalen, persönlichem Religionserlebnis.

Dieselben religiös geprägten Wertvorstellungen, die die Inhalte der elektronischen Kirche beeinflußen, erklären auch, weshalb die Kommerzialisierung von Religion im Fernsehen durch die konservativen Protestanten in keinem wirklichen Widerspruch zu ihren christlichen Prinzipien steht. Ihr pragmatisches Denken, besonders gegenüber dem technologischen Fortschritt, bedingte geradezu von Beginn an die Verwendung der neuesten Technologien im Bereich der Telekommunikation, wie das Fernsehen im allgemeinen, die Kabel- und Satellitentechnik sowie die Datenkommunikation im speziellen. Man kann die Fernsehprediger der elektronischen Kirche als Vorreiter beim Einsatz der neuen Medien im religiösen Rundfunk bezeichnen.

Die Erweckungsprediger des 19. Jahrhunderts hinterließen den Fernsehpredigern des späten 20. Jahrhunderts ein weiteres entscheidendes Vermächtnis, nämlich die institutionelle Grundlage für Massenevangelisationen. Unter der Voraussetzung der seit 1791 in der amerikanischen Verfassung verankerten Religions-, Meinungs- und Redefreiheit sowie der Garantie der Freiheit von staatlicher Kontrolle, schufen sie erstmals autarke und von Kirchenstrukturen unabhängige Parakirchen mit einer hierarchischen Rollenverteilung, die dem charismatischen Prediger die größte Macht zuteilte. Die Evangelisationskampagnen wurden zu routinierten, straff organisierten Ereignissen, deren Erfolg - d. h. der Gewinn möglichst vieler spendenbereiter Seelen - mit psychologischen, Public Relations- und Marketing- 
methoden herbeigeführt wurde. Um eine breite Öffentlichkeit anzusprechen, boten diese evangelikalen Prediger ein egalitäres Religionskonzept, welches auf theologische Reflexion zugunsten von Unterhaltung - in Form von visuellen und theatralischen Darbietungen - verzichtete. Damit reihten sie sich, wie die Televangelisten des Fernsehzeitalters nach ihnen - ebenfalls charismatische Autoritätspersonen innerhalb ihrer parakirchlichen Unternehmen -, in die Tradition der "popular culture" ein. Der einzige wesentliche Unterschied zwischen den Erweckungspredigten des 19. und der elektronischen Kirche des späten 20. Jahrhunderts ist der Einsatz modernster Kommunikationstechnologie.

Seit der zweiten Hälfte des 20. Jahrhunderts kam die „Federal Communications Commission" der elektronischen Kirche entgegen, indem sie ihr durch begünstigende Regelungen den uneingeschränkten Zugang zum kommerziellen und zum öffentlichen, nicht-kommerziellen Fernsehen verschaffte. Aus wirtschaftlichem Interesse heraus änderten schließlich auch die großen überregionalen Fernsehgesellschaften $\mathrm{CBS}, \mathrm{NBC}$ und $\mathrm{ABC}$ ihre Richtlinien zugunsten der elektronischen Kirche und zogen künftig die bezahlten Programme religiös-konservativer Gruppen denen vor, die von den Hauptkirchen auf Kosten der Fernsehanstalten produziert und gesendet wurden.

Doch die Televangelisten trugen auch selbst auf vielfältige Weise zu der marktbeherrschenden Stellung der elektronischen Kirche innerhalb des religiösen Rundfunks bei, indem sie beispielsweise ihre Programmformate und -inhalte an die Anforderungen des amerikanischen Fernsehens anpaßten und einen hohen Grad an Professionalität in der Fernsehproduktion erreichten. Die jüngste Generation der Fernsehprediger produziert weniger herkömmliche Fernsehpredigten als vielmehr unterhaltende Talk-Shows nach dem Vorbild des säkularen kommerziellen Fernsehens. Auch die Inhalte sind nicht mehr primär religiöser, sondern immer häufiger sozialer und politischer Natur. Betrachtet man diese Inhalte vor dem Hintergrund der von der elektronischen Kirche vermittelten Wertvorstellungen (wie Erfolg, Macht, Gewinnstreben, Konsum und Sicherheit, statt sozialem Engagement und Nächstenliebe), so wird deutlich, daß die von der elektronischen Kirche konstruierte soziale Realität eher mit der Realitätsdefinition des Fernsehens und weniger mit der der christlichen Religion übereinstimmt.

Die 80er Jahre, auch als „decade of destiny“ oder „Ecstatic Eighties“ bezeichnet, waren die Zeit des größten politischen und gesellschaftspolitischen Wirkens und Einflußes der elektronischen Kirche und ihrer Televangelisten, als Teil der Bewegung der Neuen Christlichen Rechten in Amerika. Die Darstellung des Zusammenspiels der religiös-konservativen Fernsehpastoren innerhalb der NCR mit der wiedererstarkten Neuen Rechten zeigt, daß die Televangelisten keine unbedeutende Randerscheinung, sondern einen ernstzunehmenden politischen Wählerblock darstellten, der sich bei den Medien und innerhalb des politischen Institutionensystems Gehör verschafft hatte. Am deutlichsten wurde dies wohl an der Präsidentschaftskandidatur des Televangelisten Pat Robertson im Wahljahr 1988.

Die Zuschaueranalyse macht deutlich, daß das Publikum der elektronischen Kirche in den 80er Jahren zwar eine relativ kleine, sehr religiöse und in erster Linie konservative Subgruppe innerhalb der amerikanischen Fernsehbevölkerung war, doch diese Zuschauer selektierten und sahen die Programme der Televangeli- 
sten gezielt. Da die von ihnen erwarteten Bedürfnisse von ihren Fernsehpredigern auch befriedigt wurden, legitimierten diese loyalen Zuschauer die Aktivitäten der elektronischen Kirche. Dies zeigte sich unter anderem in ihrem Spendenverhalten, in den Anruferzahlen bei den jeweiligen Fernsehkirchen und in ihrem Engagement in den diversen Gruppen der Neuen Christlichen Rechten.

\section{SUMMARY: The ,Electronic Church' in the United States of America}

The term ,electronic church' comprises more than just religious television as it is also produced to a lesser degree by other religious groups in America. The electronic church is above all an industry and an established institution within the American religion and television scene.

This article will give an overview of the electronic church in the United States which does not yet exist in Germany in this form. It will show in which specific way the two components ,electronic (or television) and ,church" (or religion) have contributed towards the formation and the success of this institution. The structure an organization of the American television industry and the development of religious radio broadcasting will be explained.

Furthermore, it will be shown that the television evangelists do not only want to reach a broad public via their television messages, but that they are also looking for other ways. Here, the extent of the influence of the conservative television preachers on the legislature, executive und judicature in the America of the 1980 s will be shown clearly.

In conclusion, there will be an analysis of the recipients of the television evangelists and their programmes. An account of the viewing figures of the electronic church, the demographic, sozio-economic and religious composition of its spectators and their motivation as to watching these religious television programmes is meant to make possible an estination of the influence and the effect of the electronic church.

\section{RÉSUMÉ: L'église électronique aux Etats-Unis d'Amérique}

Le terme „église électronique“ comporte plus qu'une notion de télévision religeuse, comme celles qui sont produites en petit nombre par d'autres groupes religieux en Amérique. L'église électronique est avant tout une industrie et une institution établie à l'interieur du cadre américain de la religion et de la télévision.

Cet article doit nous donner un aperçu de ce que représente l'église électronique aux Etats-Unis, une église qui, en Allemagne, n'existe pas encore sous cette forme. On va exposer de quelle façon spécifique les deux composantes „électronique“ (ou encore „télévision“) et „église“ (ou encore „religion“) ont contribué à la naissance et au succès de cette institution. On ca expliquer la structure et l'organisation de l'industrie américaine de la télévision et l'évolution de la radio religieuse.

Ensuite, on montrera que les „télévangélistes“ ne veulent pas seulement atteindre un large public par leurs message télévisés, mais qu'ils cherchent aussi d'autres chemins. On évoquera ici l'étendue de l'influence des prédicateurs conser- 
vateurs de la télévision sur le pouvoir législatif, le pouvoir exécutif et le pouvoir judiciaire en Amérique des années quatre-vingts.

L'article se termine par une analyse des auditeurs des "télévangélistes“ et de leurs émissions. Des éléments tels que l'aperçu du taux d'écoute de l'èglise électronique, la composition démographique, socio-économique et religieuse de ses téléspectateurs, ainsi que leurs motivations à regarder ces émissions religieuse télévisées, doivent permettre de mesurer l'influence de l'église électronique.

\section{RESUMEN: La Iglesia Electrónica en los Estados Unidos de América}

El concepto „iglesia electrónica“ tiene un contenido más amplio que el de televisíon religiosa, que se da también en una mínima medida entre otros grupos religiosos en América. La iglesia electrónica es, sobre todo, una industria y una institución establecida dentro del panorama de la televisión y de la religión.

Este artículo pretende dar una visión general de la iglesia electrónica de los Estados Unidos, que en esa forma no es conocida todavia en Alemania.

Se expone de qué manera específica han contribuido estos dos componentes „electrónico“ (es decir, televisión) e iglesia (es decir, religión), a la formación, creación y éxito de esta institución.

Se explica dilucida la estructura y organización de la industria televisiva americana y el desarrollo de la radiodifusión religiosa.

Adicionalmente se muestra que los tele-evangelistas no sólo quieren llegar a la opinión pública a través de sus mensajes televisivos, sino que también buscan otros caminos. Además se pone en claro la dimensión de la influencia que tuvieron los predicadores de televisión conservadores durante los años ochenta, en el campo legislativo, ejecutivo y judicial.

A ésto le sigue, por último, un análisis de los receptores de los televangelistas y de sus programas. Mirando el porcentaje de la participación de televidentes de la iglesia electrónica, la conformación demográfica, socio-económica y religio$\mathrm{sa}$, así como las motivaciones de ellos para ver estos programas, nos permite medir la influencia y las consecuencias de la iglesia electrónica. 